\title{
LARGE DEVIATIONS FOR EIGENVALUES OF SAMPLE COVARIANCE MATRICES, WITH APPLICATIONS TO MOBILE COMMUNICATION SYSTEMS
}

\author{
ANNE FEY, ${ }^{*}$ Delft University of Technology \\ REMCO VAN DER HOFSTAD, ${ }^{* *}$ Eindhoven University of Technology \\ MARTEN J. KLOK, ${ }^{* * *}$ Delft University of Technology
}

\begin{abstract}
We study sample covariance matrices of the form $\boldsymbol{W}=(1 / n) \boldsymbol{C} \boldsymbol{C}^{\top}$, where $\boldsymbol{C}$ is a $k \times n$ matrix with independent and identically distributed (i.i.d.) mean 0 entries. This is a generalization of the so-called Wishart matrices, where the entries of $\boldsymbol{C}$ are i.i.d. standard normal random variables. Such matrices arise in statistics as sample covariance matrices, and the high-dimensional case, when $k$ is large, arises in the analysis of DNA experiments. We investigate the large deviation properties of the largest and smallest eigenvalues of $\boldsymbol{W}$ when either $k$ is fixed and $n \rightarrow \infty$ or $k_{n} \rightarrow \infty$ with $k_{n}=o(n / \log \log n)$, in the case where the squares of the i.i.d. entries have finite exponential moments. Previous results, proving almost sure limits of the eigenvalues, require only finite fourth moments. Our most explicit results for large $k$ are for the case where the entries of $\boldsymbol{C}$ are \pm 1 with equal probability. We relate the large deviation rate functions of the smallest and largest eigenvalues to the rate functions for i.i.d. standard normal entries of $\boldsymbol{C}$. This case is of particular interest since it is related to the problem of decoding of a signal in a codedivision multiple-access (CDMA) system arising in mobile communication systems. In this example, $k$ is the number of users in the system and $n$ is the length of the coding sequence of each of the users. Each user transmits at the same time and uses the same frequency; the codes are used to distinguish the signals of the separate users. The results imply large deviation bounds for the probability of a bit error due to the interference of the various users.
\end{abstract}

Keywords: Sample covariance matrix; large deviation; eigenvalue; CDMA with softdecision parallel interference cancellation

2000 Mathematics Subject Classification: Primary 60F10; 62H20; 94A99

\section{Introduction}

The sample covariance matrix $\boldsymbol{W}$ of a matrix $\boldsymbol{C}$ with $k$ rows and $n$ columns is defined as $(1 / n) \boldsymbol{C} \boldsymbol{C}^{\top}$. If $\boldsymbol{C}$ has random entries then the spectrum of $\boldsymbol{W}$ is random as well. Typically, $\boldsymbol{W}$ is studied in the case that $\boldsymbol{C}$ has independent and identically distributed (i.i.d.) entries, with mean 0 and variance 1 . For this kind of $\boldsymbol{C}$, it is known that, when $k, n \rightarrow \infty$ such that $k / n=\beta$, where

\footnotetext{
Received 8 June 2007; revision received 10 November 2008.

* Postal address: DIAM, Delft University of Technology, Mekelweg 4, 2628 CD Delft, The Netherlands.

Email address: a.c.fey-denboer@tudelft.nl

** Postal address: Department of Mathematics, Eindhoven University of Technology, PO Box 513, 5600 MB

Eindhoven, The Netherlands. Email address: rhofstad@win.tue.nl

*** Current address: ORTEC BV, Orlyplein 145c, 1043 DV Amsterdam, The Netherlands.

Email address: mklok@ortec.nl
} 
$\beta$ is a constant, the eigenvalue density tends to a deterministic density [14]. The boundaries of the support of this distribution are $(1-\sqrt{\beta})_{+}^{2}$ and $(1+\sqrt{\beta})^{2}$, where $x_{+}=\max \{0, x\}$. This suggests that the smallest eigenvalue, $\lambda_{\min }$, converges to $(1-\sqrt{\beta})_{+}^{2}$, while the largest eigenvalue, $\lambda_{\max }$, converges to $(1+\sqrt{\beta})^{2}$. Bai and Yin [3] proved the almost sure convergence of $\lambda_{\min }$ to $(1-\sqrt{\beta})_{+}^{2}$. Bai et al. [4] proved the almost sure convergence of $\lambda_{\max }$ to $(1+\sqrt{\beta})^{2}$; see also [23]. The strongest results apply in the case that all the entries of $\boldsymbol{C}$ are i.i.d. with mean 0 , variance 1 , and finite fourth moment. Related results, including a central limit theorem for the linear spectral statistics, can be found in [1] and [2], to which we also refer for an overview of the extensive literature.

In the special case that the entries of $\boldsymbol{C}$ have a standard normal distribution, $\boldsymbol{W}$ is called a Wishart matrix. Wishart matrices play an important role in multivariate statistics as they describe the correlation structure in i.i.d. Gaussian multivariate data. For Wishart matrices, the large deviation rate function for the eigenvalue density with rate $1 / n^{2}$ has been derived in [10] and [12]. However, the proofs heavily depend on the fact that $\boldsymbol{C}$ has standard normal i.i.d. entries, for which the density of the ordered eigenvalues can be explicitly computed.

In this paper we investigate the large deviation rate functions with rate $1 / n$ of the smallest and largest eigenvalues of $\boldsymbol{W}$ for certain non-Gaussian entries of $\boldsymbol{C}$. We pose a strong condition on the tails of the entries, by requiring that the exponential moment of the square of the entries is bounded in a neighborhood of the origin. We will also comment on this assumption, which we believe to be necessary for our results to apply.

We let $n \rightarrow \infty$, and $k$ is either fixed or tends to $\infty$ not faster than $o(n / \log \log n)$. Our results imply that all eigenvalues tend to 1 and that all other values are large deviations. We obtain the asymptotic large deviation rate function of $\lambda_{\min }$ and $\lambda_{\max }$ when $k \rightarrow \infty$. In certain special cases we can show that the asymptotic large deviation rate function is equal to the one for Wishart matrices, which can be interpreted as saying that the spectrum of sample covariance matrices with large $k$ and $n$ is close to the one for i.i.d. standard normal entries. This proves a kind of universality result for the large deviation rate functions.

This paper is organized as follows. In Section 2 we derive an explicit expression for the large deviation rate functions of $\lambda_{\min }$ and $\lambda_{\max }$. In Section 3 we calculate lower bounds for the case in which the distribution of $C_{m i}$ is symmetric around 0 and $\left|C_{m i}\right|<M$ almost surely (a.s.) for some $M>0$. In Section 4 we specialize to the case where $C_{m i}= \pm 1$ with equal probability, which arises in an application in wireless communication. We describe the implications of our results in this application in Section 5. Part of the results for this application have been presented at an electrical engineering conference [8].

\section{General mean 0 entries of $C$}

In this section we prove large deviation results for the smallest and largest eigenvalues of sample covariance matrices.

\subsection{Large deviations for $\lambda_{\min }$ and $\lambda_{\max }$}

Define $\boldsymbol{W}=(1 / n) \boldsymbol{C} \boldsymbol{C}^{\top}$ to be the matrix of sample covariances. We denote by $\mathrm{P}$ the law of $\boldsymbol{C}$ and by $\mathrm{E}$ the corresponding expectation. Throughout the paper, we assume that the i.i.d. real matrix elements of $\boldsymbol{C}$ are normalized, i.e.

$$
\mathrm{E}\left[C_{i j}\right]=0, \quad \operatorname{var}\left(C_{i j}\right)=1
$$

The former assumption implies that, a.s., the off-diagonal elements of the matrix $\boldsymbol{W}$ converge 
to 0 , the latter assumption implies that the diagonal elements converge to 1 a.s. By a rescaling argument, the second assumption is without loss of generality.

In this subsection we rewrite the probability for a large deviation of the largest and smallest eigenvalues of $\boldsymbol{W}, \lambda_{\max }$ and $\lambda_{\min }$, respectively, into that of a large deviation of a sum of i.i.d. random variables. This rewrite allows us to use Cramér's theorem to obtain an expression for the rate function. In this subsection we give a heuristic derivation of our result, which will be turned into a proof in Subsection 2.2.

For any matrix $\boldsymbol{W}$, and any vector $\boldsymbol{x}$ with $k$ coordinates and norm $\|\boldsymbol{x}\|_{2}=1$, we have

$$
\lambda_{\min } \leq\langle\boldsymbol{x}, \boldsymbol{W} \boldsymbol{x}\rangle \leq \lambda_{\max },
$$

where $\langle\boldsymbol{x}, \boldsymbol{y}\rangle$ denotes the inner product in $\mathbb{R}^{k}$. Moreover, for the normalized eigenvector $\boldsymbol{x}_{\min }$ corresponding to $\lambda_{\min }$, the lower bound is attained, while for the normalized $\boldsymbol{x}_{\max }$ corresponding to $\lambda_{\max }$, the upper bound is attained. Therefore, we can write

$$
\begin{aligned}
& P_{\min }(\alpha)=\mathrm{P}\left(\lambda_{\min } \leq \alpha\right)=\mathrm{P}\left(\text { there exists } \boldsymbol{x}:\|\boldsymbol{x}\|_{2}=1,\langle\boldsymbol{x}, \boldsymbol{W} \boldsymbol{x}\rangle \leq \alpha\right), \\
& P_{\max }(\alpha)=\mathrm{P}\left(\lambda_{\max } \geq \alpha\right)=\mathrm{P}\left(\text { there exists } \boldsymbol{x}:\|\boldsymbol{x}\|_{2}=1,\langle\boldsymbol{x}, \boldsymbol{W} \boldsymbol{x}\rangle \geq \alpha\right) .
\end{aligned}
$$

We use the fact that $P_{\min }(\alpha)$ and $P_{\max }(\alpha)$ are the probabilities of union of events, and bound this probability from below by considering only one $\boldsymbol{x}$ and from above by summing over all $\boldsymbol{x}$. Since there are uncountably many possible $\boldsymbol{x}$, we will do this approximately by summing over a finite number of vectors. The lower bound for the probability yields an upper bound for the rate function, and vice versa.

We first heuristically explain the form of the rate functions of $\lambda_{\max }$ and $\lambda_{\min }$, and highlight the proof. The special form of a sample covariance matrix allows us to rewrite

$$
\langle\boldsymbol{x}, \boldsymbol{W} \boldsymbol{x}\rangle=\frac{1}{n}\left\|\boldsymbol{C}^{\top} \boldsymbol{x}\right\|_{2}^{2}=\frac{1}{n} \sum_{i=1}^{n}\left(\sum_{m=1}^{k} x_{m} C_{m i}\right)^{2}=\frac{1}{n} \sum_{i=1}^{n} S_{\boldsymbol{x}, i}^{2},
$$

where

$$
S_{\boldsymbol{x}, i}=\sum_{m=1}^{k} x_{m} C_{m i}
$$

with $S_{x, i}$ i.i.d. for $i=1, \ldots, m$. Define

$$
I_{k}(\alpha)=\inf _{\boldsymbol{x} \in \mathbb{R}^{k}:\|\boldsymbol{x}\|_{2}=1} \sup _{t}\left(t \alpha-\log \mathrm{E}\left[\exp \left\{t S_{\boldsymbol{x}, 1}^{2}\right\}\right]\right) .
$$

Since $\mathrm{E}\left[S_{\boldsymbol{x}, 1}^{2}\right]=1$ and $t \mapsto \log \mathrm{E}\left[\exp \left\{t S_{\boldsymbol{x}, 1}^{2}\right\}\right]$ is increasing and convex, we see that, for fixed $\boldsymbol{x}$, the optimal $t$ is nonnegative for $\alpha \geq 1$ and nonpositive for $\alpha \leq 1$. The sign of $t$ will play an important role in the proofs in Sections 3 and 4.

We can now state the first result of this paper.

Theorem 2.1. Assume that (2.1) holds. Then,

(a) for all $\alpha \geq 1$ and fixed $k \geq 2$,

$$
\limsup _{n \rightarrow \infty}-\frac{1}{n} \log \mathrm{P}\left(\lambda_{\max } \geq \alpha\right) \leq I_{k}(\alpha)
$$

and

$$
\liminf _{n \rightarrow \infty}-\frac{1}{n} \log \mathrm{P}\left(\lambda_{\max } \geq \alpha\right) \geq \lim _{\varepsilon \downarrow 0} I_{k}(\alpha-\varepsilon),
$$


(b) for all $0 \leq \alpha \leq 1$ and fixed $k \geq 2$,

$$
\limsup _{n \rightarrow \infty}-\frac{1}{n} \log \mathrm{P}\left(\lambda_{\min } \leq \alpha\right) \leq I_{k}(\alpha)
$$

and

$$
\liminf _{n \rightarrow \infty}-\frac{1}{n} \log \mathrm{P}\left(\lambda_{\min } \leq \alpha\right) \geq \lim _{\varepsilon \downarrow 0} I_{k}(\alpha+\varepsilon) .
$$

When there exists an $\varepsilon>0$ such that $\mathrm{E}\left[\exp \left\{\varepsilon C_{11}^{2}\right\}\right]<\infty$ and when $\operatorname{var}\left(C_{11}^{2}\right)>0$, then $I_{k}(\alpha)>0$ for all $\alpha \neq 1$.

We will now discuss the main result in Theorem 2.1. Theorem 2.1 is only useful when $I_{k}(\alpha)>0$, which we prove under the strong condition that there exists an $\varepsilon>0$ such that $\mathrm{E}\left[\exp \left\{\varepsilon C_{11}^{2}\right\}\right]<\infty$. For example, almost sure limits for the largest and smallest eigenvalues are proved under the much weaker condition that the fourth moment of the matrix entries $C_{i m}$ is finite. However, it is well known that the exponential bounds present in large deviations are valid only when the random variables under consideration have finite exponential moments (see, e.g. Theorem 2.2, below). In this case, the rate functions can be equal to 0 , and the large deviation results are rather uninformative. Since the eigenvalues are quadratic in the entries $\left\{C_{i m}\right\}_{i, m}$, this translates into the above condition, which we therefore believe to be necessary.

Secondly, we note that, due to the occurrence of an infimum over $\boldsymbol{x}$ and a supremum over $t$, it is unclear whether the function $\alpha \mapsto I_{k}(\alpha)$ is continuous. Clearly, when $\alpha \mapsto I_{k}(\alpha)$ is continuous, the upper and lower bounds in (2.5) and (2.6), as well as the ones in (2.7) and (2.8), are equal. We will see that this is the case for Wishart matrices in Subsection 2.3. The function $\alpha \mapsto I_{k}(\alpha)$ can easily be seen to be increasing on $[1, \infty)$ and decreasing on $(0,1]$, since $\alpha \mapsto \sup _{t}\left(t \alpha-\log \mathrm{E}\left[\exp \left\{t S_{\boldsymbol{x}, 1}^{2}\right\}\right]\right)$ has the same monotonicity properties for every fixed $\boldsymbol{x}$, so

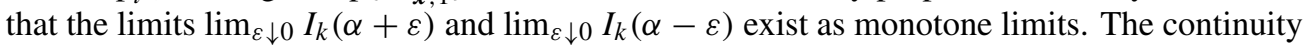
of $\alpha \mapsto I_{k}(\alpha)$ is not obvious. For example, in the simplest case where $C_{i j}= \pm 1$ with equal probability, we know that the large deviation rate function is not continuous, since the largest eigenvalue is at most $k$. Therefore, $\mathrm{P}\left(\lambda_{\max } \geq \alpha\right)=0$ for any $\alpha>k$, and if $\alpha \mapsto I_{k}(\alpha)$ is the rate function of $\lambda_{\max }$ for $\alpha \geq 1$ then $I_{k}(\alpha)=\infty$ for $\alpha>k$. It remains an interesting problem to determine in what cases $\alpha \mapsto I_{k}(\alpha)$ is continuous.

Finally, we only prove that $I_{k}(\alpha)>0$ for all $\alpha \neq 1$ when $\operatorname{var}\left(C_{11}^{2}\right)>0$. By the normalization that $\mathrm{E}\left[C_{11}\right]=0$ and $\mathrm{E}\left[C_{11}^{2}\right]=1$, this excludes only the case in which $C_{11}= \pm 1$ with equal probability. This case will be investigated in more detail in Theorem 4.1, below, where we will also prove a lower bound implying that $I_{k}(\alpha)>0$ for all $\alpha \neq 1$.

Let

$$
I_{k}(\alpha, \beta)=\inf _{\boldsymbol{x}, \boldsymbol{y} \in \mathbb{R}^{k}:\|\boldsymbol{x}\|_{2}=\|\boldsymbol{y}\|_{2}=1} \sup _{s, t}\left(t \alpha+s \beta-\log \mathrm{E}\left[\exp \left\{t S_{\boldsymbol{x}, 1}^{2}+s S_{\boldsymbol{y}, 1}^{2}\right\}\right]\right) .
$$

Our proof also reveals that, for all $0 \leq \beta \leq 1$ and $\alpha \geq 1$,

$$
\limsup _{n \rightarrow \infty}-\frac{1}{n} \log \mathrm{P}\left(\lambda_{\max } \geq \alpha, \lambda_{\min } \leq \beta\right) \geq I_{k}(\alpha, \beta)
$$

and

$$
\lim _{n \rightarrow \infty}-\frac{1}{n} \log \mathrm{P}\left(\lambda_{\max } \geq \alpha, \lambda_{\min } \leq \beta\right) \leq \lim _{\varepsilon \downarrow 0} I_{k}(\alpha+\varepsilon, \beta-\varepsilon) .
$$


For Wishart matrices, for which the entries of $\boldsymbol{C}$ are i.i.d. standard normal, the random variable $S_{\boldsymbol{x}, i}$ has a standard normal distribution, so that we can explicitly calculate $I_{k}(\alpha)$. We will elaborate on this in Subsection 2.3. For the case in which $C_{m i}= \pm 1$ with equal probability, Theorem 2.1 and its proof have also appeared in [8].

\subsection{Proof of Theorem $2.1(a)$ and (b)}

In the proof we will repeatedly make use of the largest-exponent-wins principle. We first give a short explanation of this principle. This principle concerns the exponential rate of the sum of two (or more) probabilities. From this point, we will abbreviate 'exponential rate of a probability' by 'rate'. Because of the minus sign, a smaller rate $I$ means a larger exponent and, thus, a larger probability. Thus, if, for two events $E_{1}$ and $E_{2}$, both depending on some parameter $n$, we have

$$
\mathrm{P}\left(E_{1}\right) \sim \exp \left\{-n I_{1}\right\} \quad \text { and } \mathrm{P}\left(E_{2}\right) \sim \exp \left\{-n I_{2}\right\}
$$

then

$$
-\lim _{n \rightarrow \infty} \frac{1}{n} \log \left(\mathrm{P}\left(E_{1}\right)+\mathrm{P}\left(E_{2}\right)\right) \sim \min \left\{I_{1}, I_{2}\right\} .
$$

In words, the principle states that, as $n \rightarrow \infty$, the smallest exponent (i.e. the largest rate) will become negligible. It also implies that

$$
-\lim _{n \rightarrow \infty} \frac{1}{n} \log \mathrm{P}\left(E_{1} \cup E_{2}\right) \sim \min \left\{I_{1}, I_{2}\right\} .
$$

In the proof we will make essential use of Cramér's theorem, which we state here for the sake of completeness.

Theorem 2.2. (Cramér's theorem and the Chernoff bound.) Let $\left(X_{i}\right)_{i=1}^{\infty}$ be a sequence of i.i.d. random variables. Then, for all $a \geq \mathrm{E}\left[X_{1}\right]$,

$$
-\lim _{n \rightarrow \infty} \frac{1}{n} \log \mathrm{P}\left(\frac{1}{n} \sum_{i=1}^{n} X_{i} \geq a\right)=\sup _{t \geq 0}\left(t a-\log \mathrm{E}\left[\exp \left\{t X_{1}\right\}\right]\right),
$$

while, for all $a \leq \mathrm{E}\left[X_{1}\right]$,

$$
-\lim _{n \rightarrow \infty} \frac{1}{n} \log \mathrm{P}\left(\frac{1}{n} \sum_{i=1}^{n} X_{i} \leq a\right)=\sup _{t \leq 0}\left(t a-\log \mathrm{E}\left[\exp \left\{t X_{1}\right\}\right]\right) .
$$

The upper bounds in (2.12) and (2.13) hold for every $n$. Furthermore, when $\mathrm{E}\left[\exp \left\{t X_{1}\right\}\right]<\infty$ for all $t$ with $|t| \leq \varepsilon$ and some $\varepsilon>0$, then the right-hand sides of (2.12) and (2.13) are strictly positive for all $a \neq \mathrm{E}\left[X_{1}\right]$.

See, e.g. [15, Theorem 1.1 and Proposition 1.9] for this result, and see [6] and [7] for general introductions to large deviation theory.

For the proof of Theorem 2.1(a) and (b), we start by showing that $I_{k}(\alpha)>0$ for all $\alpha \neq 1$ when there exists an $\varepsilon>0$ such that $\mathrm{E}\left[\exp \left\{\varepsilon C_{11}^{2}\right\}\right]<\infty$ and when $\operatorname{var}\left(C_{11}^{2}\right)>0$. For this, we note that, by the Cauchy-Schwarz inequality and (2.3), for every $\boldsymbol{x}$ with $\|\boldsymbol{x}\|_{2}=1$,

$$
S_{\boldsymbol{x}, i}^{2} \leq \sum_{m=1}^{k} x_{m}^{2} \sum_{m=1}^{k} C_{m i}^{2}=\sum_{m=1}^{k} C_{m i}^{2}
$$


so that $\mathrm{E}\left[\exp \left\{t S_{x, i}^{2}\right\}\right] \leq \mathrm{E}\left[\exp \left\{t C_{11}^{2}\right\}\right]^{k}<\infty$ whenever there exists an $\varepsilon>0$ such that $\mathrm{E}\left[\exp \left\{\varepsilon C_{11}^{2}\right\}\right]<\infty$. Thus, uniformly in $\boldsymbol{x}$ with $\|\boldsymbol{x}\|_{2}=1$, the random variables $S_{\boldsymbol{x}, i}^{2}$ have bounded exponential moments for $t \leq \varepsilon$. As a result, the Taylor expansion,

$$
\log \mathrm{E}\left[\exp \left\{t S_{x, i}^{2}\right\}\right]=t+\frac{t^{2}}{2} \operatorname{var}\left(S_{x, i}^{2}\right)+\mathcal{O}\left(|t|^{3}\right)
$$

holds uniformly in $\boldsymbol{x}$ with $\|\boldsymbol{x}\|_{2}=1$. We compute, since $\mathrm{E}\left[S_{\boldsymbol{x}, i}^{2}\right]=\mathrm{E}\left[C_{11}^{2}\right]=1$ and, for $\boldsymbol{x}$ with $\|x\|_{2}=1$,

$$
\mathrm{E}\left[S_{x, i}^{4}\right]=3\left(\sum_{m} x_{m}^{2}\right)^{2}-3 \sum_{m} x_{m}^{4}+\mathrm{E}\left[C_{11}^{4}\right] \sum_{m} x_{m}^{4}=3-3 \sum_{m} x_{m}^{4}+\mathrm{E}\left[C_{11}^{4}\right] \sum_{m} x_{m}^{4},
$$

that

$$
\operatorname{var}\left(S_{x, i}^{2}\right)=3-3 \sum_{m} x_{m}^{4}+\mathrm{E}\left[C_{11}^{4}\right] \sum_{m} x_{m}^{4}-1=2-2 \sum_{m} x_{m}^{4}+\operatorname{var}\left(C_{11}^{2}\right) \sum_{m} x_{m}^{4},
$$

which is bounded, since, by assumption, $\mathrm{E}\left[\exp \left\{t C_{11}^{2}\right\}\right]<\infty$. Furthermore, $\sum_{m} x_{m}^{4} \in[0,1]$ uniformly in $\boldsymbol{x}$ with $\|\boldsymbol{x}\|_{2}=1$, so that, again uniformly in $\boldsymbol{x}$ with $\|\boldsymbol{x}\|_{2}=1, \operatorname{var}\left(S_{\boldsymbol{x}, i}^{2}\right) \geq$ $\min \left\{2, \operatorname{var}\left(C_{11}^{2}\right)\right\}>0$. We conclude that, for sufficiently small $t$, uniformly in $\boldsymbol{x}$ with $\|\boldsymbol{x}\|_{2}=1$, and by ignoring higher-order Taylor expansion terms of $t \mapsto \log \mathrm{E}\left[\exp \left\{t S_{\boldsymbol{x}, i}^{2}\right\}\right]$ in (2.14), which is allowed when $|t|$ is sufficiently small,

$$
\log \mathrm{E}\left[\exp \left\{t S_{x, i}^{2}\right\}\right] \leq t+t^{2} \min \left\{2, \operatorname{var}\left(C_{11}^{2}\right)\right\} .
$$

In turn, this implies that, for $|t| \leq \varepsilon$ small, and uniformly in $\boldsymbol{x}$ with $\|\boldsymbol{x}\|_{2}=1$,

$$
\begin{aligned}
I_{k}(\alpha) & \geq \inf _{\boldsymbol{x} \in \mathbb{R}^{k}:\|\boldsymbol{x}\|_{2}=1} \sup _{|t| \leq \varepsilon}\left(t \alpha-\log \mathrm{E}\left[\exp \left\{t S_{\boldsymbol{x}, 1}^{2}\right\}\right]\right) \\
& \geq \inf _{\boldsymbol{x} \in \mathbb{R}^{k}:\|\boldsymbol{x}\|_{2}=1} \sup _{|t| \leq \varepsilon}\left(t(\alpha-1)-\frac{t^{2}}{2} \min \left\{2, \operatorname{var}\left(C_{11}^{2}\right)\right\}\right)>0
\end{aligned}
$$

the latter bound holding for every $\alpha \neq 1$ when $\operatorname{var}\left(C_{11}^{2}\right)>0$. This completes the proof that $I_{k}(\alpha)>0$ for all $\alpha \neq 1$ when there exists an $\varepsilon>0$ such that $\operatorname{E}\left[\exp \left\{{ }_{\varepsilon} C_{11}^{2}\right\}\right]<\infty$ and $\operatorname{var}\left(C_{11}^{2}\right)>0$.

We continue by proving (2.5)-(2.8). The proof for $\lambda_{\max }$ is similar to the one for $\lambda_{\min }$, so we will focus on the latter. To obtain the upper bound of the rate of (2.2), we use the fact that, for any $\boldsymbol{x}^{\prime}$ with $\left\|\boldsymbol{x}^{\prime}\right\|_{2}=1$,

$$
\mathrm{P}\left(\lambda_{\min } \leq \alpha\right)=\mathrm{P}(\text { there exists } \boldsymbol{x}:\langle\boldsymbol{x}, \boldsymbol{W} \boldsymbol{x}\rangle \leq \alpha) \geq \mathrm{P}\left(\left\langle\boldsymbol{x}^{\prime}, \boldsymbol{W} \boldsymbol{x}^{\prime}\right\rangle \leq \alpha\right) .
$$

Now insert (2.2). Since $\boldsymbol{x}^{\prime}$ is fixed, the $S_{\boldsymbol{x}^{\prime}, i}^{2}$ are i.i.d. variables, and we can apply Cramér's theorem to obtain the upper bound for the rate function for fixed $\boldsymbol{x}^{\prime}$. This yields, for every $\boldsymbol{x}^{\prime}$,

$$
-\liminf _{n \rightarrow \infty} \frac{1}{n} \log \mathrm{P}\left(\lambda_{\min } \leq \alpha\right) \leq \sup _{t}\left(t \alpha-\log \mathrm{E}\left[\exp \left\{t S_{x^{\prime}, 1}^{2}\right\}\right]\right) .
$$

If we maximize the right-hand side over $\boldsymbol{x}^{\prime}$ then we arrive at $I_{k}(\alpha)$ as the upper bound, and we have proved (2.7). The proof of (2.5) is identical. 
We are left to prove the lower bounds in (2.6) and (2.8). For this, we wish to sum over all possible $\boldsymbol{x}$. We approximate the sphere $\|\boldsymbol{x}\|_{2}=1$ by a finite set of vectors $\boldsymbol{x}^{(j)}$ with $\left\|\boldsymbol{x}^{(j)}\right\|_{2}=1$, such that the distance between two of these vectors is at most $d$, and observe that

$$
\begin{aligned}
\left|\langle\boldsymbol{x}, \boldsymbol{W} \boldsymbol{x}\rangle-\left\langle\boldsymbol{x}^{(j)}, \boldsymbol{W} \boldsymbol{x}^{(j)}\right\rangle\right| & =\left|\left\langle\left(\boldsymbol{x}-\boldsymbol{x}^{(j)}\right), \boldsymbol{W} \boldsymbol{x}\right\rangle+\left\langle\boldsymbol{x}^{(j)}, \boldsymbol{W}\left(\boldsymbol{x}-\boldsymbol{x}^{(j)}\right)\right\rangle\right| \\
& =\left|\left\langle\boldsymbol{x}, \boldsymbol{W}\left(\boldsymbol{x}-\boldsymbol{x}^{(j)}\right)\right\rangle+\left\langle\boldsymbol{x}^{(j)}, \boldsymbol{W}\left(\boldsymbol{x}-\boldsymbol{x}^{(j)}\right)\right\rangle\right| \\
& \leq\left(\|\boldsymbol{x}\|+\left\|\boldsymbol{x}^{(j)}\right\|\right)\|\boldsymbol{W}\|\left\|\boldsymbol{x}-\boldsymbol{x}^{(j)}\right\| \\
& \leq 2 \lambda_{\max } d .
\end{aligned}
$$

We need $\lambda_{\max } \leq \kappa k$, where $\kappa$ is some large enough constant, with sufficiently high probability, which we will prove first. We have $\lambda_{\max } \leq T_{\boldsymbol{W}}$, where $T_{\boldsymbol{W}}$ is the trace of $\boldsymbol{W}$, since $\boldsymbol{W}$ is nonnegative. Note that

$$
T_{\boldsymbol{W}}=\frac{1}{n} \sum_{i=1}^{n} \sum_{m=1}^{k} C_{m i}^{2} .
$$

Thus, $T_{W}$ is a sum of $n k$ i.i.d. variables.

Since $\mathrm{E}\left[\exp \left\{t C_{11}^{2}\right\}\right]<\infty$ for all $t \leq \varepsilon$, we can use Cramér's theorem for $T_{W}$. Therefore, for any $\kappa$, by the Chernoff bound,

$$
\mathrm{P}\left(T_{W}>\kappa k\right) \leq \exp \left\{-n k I_{C^{2}}(\kappa)\right\},
$$

where

$$
I_{C^{2}}(a)=\sup _{t}\left(t a-\log \mathrm{E}\left[\exp \left\{t C_{11}^{2}\right\}\right]\right) .
$$

Since $\mathrm{E}\left[C_{11}^{2}\right]=\operatorname{var}\left(C_{11}\right)=1$, we have $I_{C^{2}}(\kappa)>0$ for any $\kappa>1$. Therefore, by picking $\kappa>1$ large enough, we can make $k I_{C^{2}}(\kappa)$ arbitrarily large. If we take $k I_{C^{2}}(\kappa)$ larger than $I_{k}(\alpha-\varepsilon)$, according to (2.11), this will not influence the result. (Note that, when $I_{k}(\alpha-\varepsilon)=\infty$ for all $\varepsilon>0$, then we can also let $k I_{C^{2}}(\kappa)$ tend to $\infty$ by taking $\kappa \rightarrow \infty$.)

It follows that

$$
\begin{aligned}
\mathrm{P}\left(\lambda_{\min } \leq \alpha\right) & \leq \mathrm{P}\left(\text { there exists } \boldsymbol{x}^{(j)}:\left\langle\boldsymbol{x}^{(j)}, \boldsymbol{W} \boldsymbol{x}^{(j)}\right\rangle \leq \alpha+2 d \kappa k\right)+\mathrm{P}\left(T_{\boldsymbol{W}}>\kappa k\right) \\
& \leq \sum_{j} \mathrm{P}\left(\left\langle\boldsymbol{x}^{(j)}, \boldsymbol{W} \boldsymbol{x}^{(j)}\right\rangle \leq \alpha+2 d \kappa k\right)+\mathrm{P}\left(T_{\boldsymbol{W}}>\kappa k\right) \\
& \leq N_{d} \sup _{\boldsymbol{x}^{(j)}} \mathrm{P}\left(\left\langle\boldsymbol{x}^{(j)}, \boldsymbol{W} \boldsymbol{x}^{(j)}\right\rangle \leq \alpha+2 d \kappa k\right)+\mathrm{P}\left(T_{\boldsymbol{W}}>\kappa k\right),
\end{aligned}
$$

where $N_{d}$ is the number of vectors in the finite approximation of the sphere. The above bound is valid for every choice of $\kappa, k, \alpha$, and $d$.

We write $\varepsilon=2 d \kappa k$, and we will later let $\varepsilon \downarrow 0$. Then, applying the largest-exponent-wins principle for $\kappa>0$ large enough, as well as Cramér's theorem together with (2.2), we arrive at

$$
\begin{aligned}
-\limsup _{n \rightarrow \infty} \frac{1}{n} \log \mathrm{P}\left(\lambda_{\min } \leq \alpha\right) \geq & \inf _{\boldsymbol{x}^{(j)}} \sup _{t}\left(t(\alpha+\varepsilon)-\log \mathrm{E}\left[\exp \left\{t S_{\boldsymbol{x}^{(j)}, 1}^{2}\right\}\right]\right) \\
& +\liminf _{n \rightarrow \infty} \frac{1}{n} \log N_{d} \\
\geq & I_{k}(\alpha+\varepsilon)+\liminf _{n \rightarrow \infty} \frac{1}{n} \log N_{d} .
\end{aligned}
$$


In a similar way, we obtain

$$
\begin{aligned}
-\limsup _{n \rightarrow \infty} \frac{1}{n} \log \mathrm{P}\left(\lambda_{\max } \geq \alpha\right) \geq & \inf _{\boldsymbol{x}^{(j)}} \sup _{t}\left(t(\alpha-\varepsilon)-\log \mathrm{E}\left[\exp \left\{t S_{\boldsymbol{x}^{(j)}, 1}^{2}\right\}\right]\right) \\
& +\liminf _{n \rightarrow \infty} \frac{1}{n} \log N_{d} \\
\geq & I_{k}(\alpha-\varepsilon)+\liminf _{n \rightarrow \infty} \frac{1}{n} \log N_{d}
\end{aligned}
$$

where we take $d$ so small that $\alpha-\varepsilon>0$.

A simple overestimation of $N_{d}$ is obtained by first taking $[-1,1]^{k} \subset \mathbb{R}^{k}$ around the origin, and laying a grid on this cube with grid length $1 / L$. We then normalize the centers of these cubes to have norm 1 . The finite set of vectors consists of the centers of the small cubes of width $2 / L$. In this case,

$$
d \leq \frac{3 \sqrt{k}}{L} \quad \text { and } \quad N_{d} \leq L^{k} .
$$

Indeed, the first bound follows since, for any vector $\boldsymbol{x}$, there exists a center of a small cube for which all coordinates are at most $1 / L$ away. Therefore, the distance to this center is at most $\sqrt{k} / L$. Since $\boldsymbol{x}$ has norm 1 , the norm of the center of the cube is between $1-\sqrt{k} / L$ and $1+\sqrt{k} / L$, and we find that the distance of $\boldsymbol{x}$ to the normalized center of the small cube is at most

$$
d \leq \frac{\sqrt{k}}{L}+\frac{\sqrt{k} / L}{1-\sqrt{k} / L} \leq 3 \frac{\sqrt{k}}{L},
$$

when $\sqrt{k} / L \leq \frac{1}{2}$. For this choice, we have $\varepsilon=6 \kappa k^{3 / 2} / L$, which we can make small by taking $L$ large.

We conclude that, for any $L<\infty, \lim _{n \rightarrow \infty}(1 / n) \log N_{d}=0$, so that, for any $\kappa>1$ sufficiently large,

$$
-\limsup _{n \rightarrow \infty} \frac{1}{n} \log \mathrm{P}\left(\lambda_{\min } \leq \alpha\right) \geq I_{k}(\alpha+\varepsilon)
$$

and

$$
-\limsup _{n \rightarrow \infty} \frac{1}{n} \log \mathrm{P}\left(\lambda_{\max } \geq \alpha\right) \geq I_{k}(\alpha-\varepsilon)
$$

when the right-hand sides are finite. Since the above statement is true for any $\varepsilon$, we can take $\varepsilon \downarrow 0$ by letting $L \uparrow \infty$. When the right-hand sides are infinite, we conclude that the left-hand sides can also be made arbitrarily large by letting $L \uparrow \infty$. This completes the proof of (2.6) and (2.8).

To prove (2.9) and (2.10), we follow the above proof. We first note that the eigenvectors corresponding to $\lambda_{\max }$ and $\lambda_{\min }$ are orthogonal. Therefore, we obtain

$$
\begin{gathered}
\mathrm{P}\left(\lambda_{\max } \geq \alpha, \lambda_{\min } \leq \beta\right)=\mathrm{P}\left(\text { there exists } \boldsymbol{x}, \boldsymbol{y}:\|\boldsymbol{x}\|_{2}=\|\boldsymbol{y}\|_{2}=1,\langle\boldsymbol{x}, \boldsymbol{y}\rangle=0,\right. \\
\langle\boldsymbol{x}, \boldsymbol{W} \boldsymbol{x}\rangle \geq \alpha,\langle\boldsymbol{y}, \boldsymbol{W} \boldsymbol{y}\rangle \leq \beta) .
\end{gathered}
$$

We now proceed as above, and for the lower bound, we pick any $\boldsymbol{x}$ and $\boldsymbol{y}$ satisfying the requirements in the probability on the right-hand side. The upper bound is slightly harder. For this, we need to pick a finite approximation for the choices of $\boldsymbol{x}$ and $\boldsymbol{y}$ such that $\|\boldsymbol{x}\|_{2}=\|\boldsymbol{y}\|_{2}=1$ and $\langle\boldsymbol{x}, \boldsymbol{y}\rangle=0$. We will now show that we can do this in such a way that the total number of pairs $\left\{\boldsymbol{x}^{(i)}, \boldsymbol{y}^{(i, j)}\right\}_{i, j \geq 1}$ is bounded by $N_{d}^{2}$, where $N_{d}$ is as in (2.17). 
We pick $\left\{\boldsymbol{x}^{(i)}\right\}_{i \geq 1}$, as in the above proof. Then, for fixed $\boldsymbol{x}^{(i)}$, we define a finite number of $\boldsymbol{y}$ such that $\left\langle\boldsymbol{x}^{(i)}, \boldsymbol{y}\right\rangle=0$. For this, we consider, for fixed $\boldsymbol{x}^{(i)}$, only those cubes of width $1 / L$ around an $\boldsymbol{x}^{(j)}$ for some $j$ that contain at least one element $z$ having norm 1 and such that $\left\langle\boldsymbol{z}, \boldsymbol{x}^{(i)}\right\rangle=0$. Fix one such cube. If there are more such $\boldsymbol{z}$ in this cube around $\boldsymbol{x}^{(j)}$ then we pick the unique element that is closest to $\boldsymbol{x}^{(j)}$. We denote this element by $\boldsymbol{y}^{(j, i)}$. The set of these elements $\boldsymbol{y}^{(j, i)}$ will be denoted by $\left\{\boldsymbol{y}^{(j, i)}\right\}_{i \geq 1}$. The finite subset of the set $\|\boldsymbol{x}\|_{2}=\|\boldsymbol{y}\|_{2}=1$ and $\langle\boldsymbol{x}, \boldsymbol{y}\rangle=0$ then consists of $\left\{\boldsymbol{x}^{(i)}, \boldsymbol{y}^{(i, j)}\right\}_{i, j \geq 1}$.

Clearly, every $\boldsymbol{x}$ and $\boldsymbol{y}$ with $\|\boldsymbol{x}\|_{2}=\|\boldsymbol{y}\|_{2}=1$ and $\langle\boldsymbol{x}, \boldsymbol{y}\rangle=0$ can be approximated by a pair $\boldsymbol{x}^{(j)}$ and $\boldsymbol{y}^{(j, i)}$ such that $\left\|\boldsymbol{x}-\boldsymbol{x}^{(j)}\right\|_{2} \leq d$ and $\left\|\boldsymbol{y}-\boldsymbol{y}^{(i, j)}\right\|_{2} \leq 2 d$. Then we can complete the proof as above.

\subsection{Special case: Wishart matrices}

To give an example, we consider Wishart matrices, for which $C_{i j}$ are i.i.d. standard normal. In this case we can compute $I_{k}(\alpha)$ and $I_{k}(\alpha, \beta)$ explicitly. To compute $I_{k}(\alpha)$, we note that, for any $\boldsymbol{x}$ such that $\|\boldsymbol{x}\|_{2}=1, S_{\boldsymbol{x}, 1}$ is standard normal. Therefore,

$$
\mathrm{E}\left[\exp \left\{t S_{\boldsymbol{x}, 1}^{2}\right\}\right]=\frac{1}{\sqrt{1-2 t}},
$$

so that

$$
I_{k}(\alpha)=\sup _{t}\left(t \alpha-\log \left(\frac{1}{\sqrt{1-2 t}}\right)\right) .
$$

In order to compute $I_{k}(\alpha)$, we note that the maximization problem over $t$ in $\sup _{t}(t \alpha-$ $\log (1 / \sqrt{1-2 t})$ is straightforward, and yields $t^{*}=\frac{1}{2}-1 / 2 \alpha$ and $I_{k}(\alpha)=\frac{1}{2}(\alpha-1-\log \alpha)$. Note that $I_{k}(\alpha)$ is independent of $k$. In particular, we see that $\alpha \mapsto I_{k}(\alpha)$ is continuous, which leads us to the following corollary.

Corollary 2.1. Let $C_{i j}$ be independent standard normals. Then,

(a) for all $\alpha \geq 1$ and fixed $k \geq 2$,

$$
\lim _{n \rightarrow \infty}-\frac{1}{n} \log \mathrm{P}\left(\lambda_{\max } \geq \alpha\right)=\frac{1}{2}(\alpha-1-\log \alpha),
$$

(b) for all $0 \leq \alpha \leq 1$ and fixed $k \geq 2$,

$$
\limsup _{n \rightarrow \infty}-\frac{1}{n} \log \mathrm{P}\left(\lambda_{\min } \leq \alpha\right)=\frac{1}{2}(\alpha-1-\log \alpha) .
$$

We next turn to the computation of $I_{k}(\alpha, \beta)$. When $\boldsymbol{x}$ and $\boldsymbol{y}$ are such that $\|\boldsymbol{x}\|_{2}=\|\boldsymbol{y}\|_{2}=1$ and $\langle\boldsymbol{x}, \boldsymbol{y}\rangle=0$, then $\left(S_{\boldsymbol{x}, 1}, S_{\boldsymbol{y}, 1}\right)$ are normally distributed. It can be easily seen that $\mathrm{E}\left[S_{\boldsymbol{x}, 1}\right]=0$ and $\mathrm{E}\left[S_{\boldsymbol{x}, 1}^{2}\right]=\|\boldsymbol{x}\|_{2}^{2}=1$, so that $S_{\boldsymbol{x}, 1}$ and $S_{\boldsymbol{y}, 1}$ are standard normal. Moreover, E $\left[S_{\boldsymbol{x}, 1} S_{\boldsymbol{y}, 1}\right]=$ $\langle\boldsymbol{x}, \boldsymbol{y}\rangle=0$, so that $\left(S_{\boldsymbol{x}, 1}, S_{\boldsymbol{y}, 1}\right)$ are in fact independent standard normal random variables. Therefore,

$$
\mathrm{E}\left[\exp \left\{t S_{\boldsymbol{x}, 1}^{2}+s S_{\boldsymbol{y}, 1}^{2}\right\}\right]=\mathrm{E}\left[\exp \left\{t S_{\boldsymbol{x}, 1}^{2}\right\}\right] \mathrm{E}\left[\exp \left\{s S_{\boldsymbol{y}, 1}^{2}\right\}\right]=\frac{1}{\sqrt{1-2 t}} \frac{1}{\sqrt{1-2 s}}
$$

and, for $\alpha \in[0,1]$ and $\beta \geq 1$,

$$
I_{k}(\alpha, \beta)=\sup _{s, t}\left(t \alpha+s \beta-\log \left(\frac{1}{\sqrt{1-2 t}}\right)-\log \left(\frac{1}{\sqrt{1-2 s}}\right)\right)=I_{k}(\alpha)+I_{k}(\beta),
$$


so that the exponential rate of the probability that $\lambda_{\max } \geq \alpha$ and $\lambda_{\min } \leq \beta$ is equal to the exponential rate of the product of the probabilities that $\lambda_{\max } \geq \alpha$ and $\lambda_{\min } \leq \beta$. This remarkable form of independence seems to be true only for Wishart matrices.

The above considerations lead to the following corollary.

Corollary 2.2. Let $C_{i j}$ be independent standard normals. Then,

$$
\lim _{n \rightarrow \infty}-\frac{1}{n} \log \mathrm{P}\left(\lambda_{\max } \geq \alpha, \lambda_{\min } \leq \beta\right)=\frac{1}{2}(\alpha-1-\log \alpha)+\frac{1}{2}(\beta-1-\log \beta) .
$$

In the sequel we will, among other things, investigate cases where, for $k \rightarrow \infty$, the rate function $I_{k}(\alpha)$ for general $C_{i j}$ converges to the Gaussian limit $I_{\infty}(\alpha)=\frac{1}{2}(\alpha-1-\log \alpha)$.

\section{Asymptotics for the eigenvalues for symmetric and bounded entries of $C$}

In this section we investigate the case where $C_{m i}$ is symmetric around 0 and $\left|C_{m i}\right|<M<\infty$ a.s., or $C_{m i}$ is standard normal. To emphasize the role of $k$, we will denote the law of $\boldsymbol{W}$ for a given $k$ by $\mathrm{P}_{k}$. We define the extension to $k=\infty$ of $I_{k}(\alpha)$ to be

$$
I_{\infty}(\alpha)=\inf _{\boldsymbol{x} \in \ell^{2}(\mathbb{N}):\|x\|_{2}=1} \sup _{t}\left(t \alpha-\log \mathrm{E}\left[\exp \left\{t S_{x, 1}^{2}\right\}\right]\right),
$$

where $\ell^{2}(\mathbb{N})$ is the space of all infinite square-summable sequences with norm

$$
\|x\|_{2}=\sqrt{\sum_{i=1}^{\infty} x_{i}^{2}}
$$

The main result in this section is the following theorem.

Theorem 3.1. Suppose that $C_{m i}$ is symmetric around 0 and that $\left|C_{m i}\right|<M<\infty$ a.s., or $C_{m i}$ is standard normal. Then, for all $k_{n} \rightarrow \infty$ such that $k_{n}=o(n / \log \log n)$,

(a) for all $\alpha \geq 1$,

$$
\liminf _{n \rightarrow \infty}-\frac{1}{n} \log \mathrm{P}_{k_{n}}\left(\lambda_{\max } \geq \alpha\right) \leq I_{\infty}(\alpha)
$$

and

$$
\limsup _{n \rightarrow \infty}-\frac{1}{n} \log \mathrm{P}_{k_{n}}\left(\lambda_{\max } \geq \alpha\right) \geq \lim _{\varepsilon \downarrow 0} I_{\infty}(\alpha-\varepsilon),
$$

(b) for all $0<\alpha \leq 1$,

$$
\liminf _{n \rightarrow \infty}-\frac{1}{n} \log \mathrm{P}_{k_{n}}\left(\lambda_{\min } \leq \alpha\right) \leq I_{\infty}(\alpha)
$$

and

$$
\liminf _{n \rightarrow \infty}-\frac{1}{n} \log \mathrm{P}_{k_{n}}\left(\lambda_{\min } \leq \alpha\right) \geq \lim _{\varepsilon \downarrow 0} I_{\infty}(\alpha+\varepsilon) .
$$

A version of this result has also been published in a conference proceeding [8] for the special case $C_{m i}= \pm 1$, each with probability $\frac{1}{2}$, and where the restriction on $k_{n}$ was $k_{n}=\mathcal{O}(n / \log n)$. Unfortunately, there is a technical error in the proof; we present the corrected proof below. In order to do so, we will rely on explicit lower bounds for $I_{k}(\alpha), \alpha \geq 1$.

A priori, it is not obvious that the limit $I_{\infty}(\alpha)$ is strictly positive for $\alpha \neq 1$. However, in the examples we will investigate later on, such as $C_{m i}= \pm 1$ with equal probability, we will see that indeed $I_{\infty}(\alpha)>0$ for $\alpha \neq 1$. Possibly, such a result can be shown more generally.

The following proposition is instrumental in the proof of Theorem 3.1. 
Proposition 3.1. Assume that $C_{m i}$ is symmetric around 0 and that $\left|C_{m i}\right|<M<\infty$ a.s., or $C_{m i}$ is standard normal. Then, for all $k, \alpha \geq M^{2}$, and $\boldsymbol{x}$ with $\|\boldsymbol{x}\|_{2}=1$,

$$
\mathrm{P}_{k}(\langle\boldsymbol{x}, \boldsymbol{W} \boldsymbol{x}\rangle \geq \alpha) \leq \exp \left\{-n J_{k}(\alpha)\right\},
$$

where

$$
J_{k}(\alpha)=\frac{1}{2}\left(\frac{\alpha}{M^{2}}-1-\log \frac{\alpha}{M^{2}}\right) .
$$

In the case where $C_{m i}= \pm 1$, for which $M>1$, we will present an improved version of this bound, valid when $\alpha \geq \frac{1}{2}$, in Theorem 4.1, below.

\subsection{Proof of Proposition 3.1}

Throughout this proof, we fix $\boldsymbol{x}$ with $\|\boldsymbol{x}\|_{2}=1$. We use (2.2) to bound, for every $t \geq 0$ and $k \in \mathbb{N}$, by the Markov inequality,

$$
\mathrm{P}_{k}(\langle\boldsymbol{x}, \boldsymbol{W} \boldsymbol{x}\rangle \geq \alpha)=\mathrm{P}_{k}\left(\exp \left\{t \sum_{i=1}^{n} S_{\boldsymbol{x}, i}^{2}\right\} \geq \mathrm{e}^{n t \alpha}\right) \leq \exp \left\{-n\left(\alpha t-\log \mathrm{E}_{k_{n}}\left[\exp \left\{t S_{\boldsymbol{x}, 1}^{2}\right\}\right]\right)\right\} .
$$

We claim that, for all $0 \leq t \leq 1 / M^{2}$,

$$
\mathrm{E}_{k_{n}}\left[\exp \left\{t S_{x, i}^{2}\right\}\right] \leq \frac{1}{\sqrt{1-2 M^{2} t}} .
$$

In the case of Wishart matrices, for which $S_{\boldsymbol{x}, i}$ has a standard normal distribution, (3.6) holds with equality for $M=1$.

We first note that (3.6) is proven in [18, Section IV] for the case in which $C_{i j}= \pm 1$ with equal probability. For any $k$ and $\boldsymbol{x}$, the bound is even valid for all $-\frac{1}{2} \leq t \leq \frac{1}{2}$. We now extend the case where $C_{i j}= \pm 1$ to the case where $C_{i j}$ is symmetric around 0 and satisfies $\left|C_{i j}\right|<M$ a.s.

We write $C_{i j}=A_{i j} C_{i j}^{*}$, where $A_{i j}=\left|C_{i j}\right|<M$ a.s. and $C_{i j}^{*}=\operatorname{sgn}\left(C_{i j}\right)$. Moreover, $A_{i j}$ and $C_{i j}^{*}$ are independent, since $C_{i j}$ has a symmetric distribution around 0 . Thus, we obtain $S_{\boldsymbol{x}, i}=S_{A_{i} \boldsymbol{x}, i}^{*}$, where $\left(A_{i} \boldsymbol{x}\right)_{j}=A_{i j} x_{j}$, and

$$
S_{\boldsymbol{y}, i}^{*}=\sum_{j=1}^{k} C_{i j}^{*} y_{j}
$$

For $S_{\boldsymbol{y}, i}^{*}$, we know that (3.6) is proven. Therefore,

$$
\mathrm{E}_{k}\left[\exp \left\{t S_{A_{i} \boldsymbol{x}, i}^{2}\right\}\right] \leq \mathrm{E}_{k}\left[\frac{1}{\sqrt{1-2 t\left\|A_{i} \boldsymbol{x}\right\|_{2}^{2}}}\right]
$$

for all $t$ such that $-\frac{1}{2} \leq t\left\|A_{i} x\right\|_{2} \leq \frac{1}{2}$ a.s. When $\|\boldsymbol{x}\|_{2}^{2}=1$, we have

$$
0 \leq\left\|A_{i} \boldsymbol{x}\right\|_{2}<M \quad \text { a.s. }
$$

Therefore,

$$
\mathrm{E}_{k}\left[\exp \left\{t S_{A_{i} x, i}^{2}\right\}\right] \leq \frac{1}{\sqrt{1-2 M^{2} t\|\boldsymbol{x}\|_{2}^{2}}} \text { for all } 0 \leq t M^{2}\|\boldsymbol{x}\|_{2}^{2} \leq \frac{1}{2}
$$


Thus, we arrive at

$$
\mathrm{P}_{k}(\langle\boldsymbol{x}, \boldsymbol{W} \boldsymbol{x}\rangle \geq \alpha) \leq \exp \left\{-n\left(\sup _{0 \leq t \leq 1 / M^{2}}\left(t \alpha-\log \frac{1}{\sqrt{1-2 M^{2} t}}\right)\right)\right\} .
$$

Note that, since $\|\boldsymbol{x}\|_{2}=1$, the bound is independent of $\boldsymbol{x}$. Taking the maximum over $t$ of the right-hand side of (3.7) yields $t^{*}=1 / 2 M^{2}-1 / 2 \alpha$, and inserting this value of $t^{*}$ into the right-hand side of (3.7) gives the result.

\subsection{Proof of Theorem 3.1}

The proof is similar to that of Theorem 2.1. For the proofs of (3.2) and (3.4), we again use (2.15), but now choose an $\boldsymbol{x}^{\prime}$ of which only the first $k$ components are nonzero. This leads to, using the fact that $k_{n} \rightarrow \infty$, so that $k_{n} \geq k$ for sufficiently large $n$,

$$
\liminf _{n \rightarrow \infty}-\frac{1}{n} \log \mathrm{P}_{k_{n}}\left(\lambda_{\max } \geq \alpha\right) \leq \sup _{t}\left(t \alpha-\log \mathrm{E}\left[\exp \left\{t S_{x^{\prime}, 1}^{2}\right\}\right]\right) .
$$

Maximizing over all $\boldsymbol{x}^{\prime}$ of which only the first $k$ components are nonzero leads to

$$
\liminf _{n \rightarrow \infty}-\frac{1}{n} \log \mathrm{P}_{k_{n}}\left(\lambda_{\max } \geq \alpha\right) \leq I_{k}(\alpha),
$$

where this bound is valid for all $k \in \mathbb{N}$. We next claim that

$$
\lim _{k \rightarrow \infty} I_{k}(\alpha)=I_{\infty}(\alpha)
$$

For this, we first note that the sequence $k \mapsto I_{k}(\alpha)$ is nonincreasing and nonnegative, so that it has a pointwise limit. Secondly, $I_{k}(\alpha) \geq I_{\infty}(\alpha)$ for all $k$, since the number of possible choices of $\boldsymbol{x}$ in (3.1) is larger than the one in (2.4). Now it is not hard to see that $\lim _{k \rightarrow \infty} I_{k}(\alpha)=I_{\infty}(\alpha)$, by splitting into the two cases depending on whether the infimum over $\boldsymbol{x}$ in (3.1) is attained or not. This completes the proof of (3.2) and (3.4).

For the proof of (3.3) and (3.5), we adapt the proof of (2.6) and (2.8). As in the proof of Theorem 2.1(a)-(b), we wish to show that the terms $(1 / n) \log N_{d}$ and $2 d \lambda_{\max }$ vanish when we take the logarithm of (2.16), divide by $n$, and let $n \rightarrow \infty$. However, this time we wish to let $k_{n} \rightarrow \infty$ as well, for $k_{n}$ as large as possible. We will have $k_{n}=o(n)$ in mind.

The overestimation (2.17) can be improved using an upper bound for the number $M_{R}=N_{1 / R}$ of spheres of radius $1 / R$ needed to cover the surface of a $k$-dimensional sphere of radius 1 [17]: when $k \rightarrow \infty$,

$$
M_{R}=4 k \sqrt{k} R^{k}(\log k+\log \log k+\log R)\left(1+\mathcal{O}\left(\frac{1}{\log k}\right)\right) \equiv f(k, R) R^{k} .
$$

This bound is valid for $R>\sqrt{k /(k-1)}$. Since we use small spheres this time, $d \leq 1 / R$.

We can also improve the upper bound for $\lambda_{\max }$. For any $\Omega_{n}>1$, which we will choose appropriately later on, we split

$$
\begin{aligned}
& P_{\min }(\alpha) \leq \mathrm{P}\left(\lambda_{\min } \leq \alpha, \lambda_{\max } \leq \Omega_{n}\right)+P_{\max }\left(\Omega_{n}\right), \\
& P_{\max }(\alpha)=\mathrm{P}\left(\alpha \leq \lambda_{\max } \leq \Omega_{n}\right)+P_{\max }\left(\Omega_{n}\right) .
\end{aligned}
$$

We first give a sketch of the proof, omitting the details. The idea is that the first term of these expressions will yield the rate function $I_{\infty}(\alpha)$. The term $P_{\max }\left(\Omega_{n}\right)$ has an exponential 
rate which is $\mathcal{O}\left(\Omega_{n}\right)-\mathcal{O}\left(k_{n} \log k_{n} / n\right)$, and, since $k_{n} \log k_{n} / n=o(\log n)$, can thus be made arbitrarily large by taking $\Omega_{n}=K \log n$ with $K>1$ large enough. This means that we can choose $\Omega_{n}$ large enough to make this rate disappear according to the largest-exponent-wins principle, (2.11). We will need different choices of $R$ for the two terms. We will now give the details of the proof.

We first bound $P_{\max }\left(\Omega_{n}\right)$ in (3.10) using (2.16). In (2.16) we choose $\kappa=M^{2}$. This leads to

$$
P_{\max }\left(\Omega_{n}\right) \leq M_{R} \sup _{\boldsymbol{x}} \mathrm{P}\left(\langle\boldsymbol{x}, \boldsymbol{W} \boldsymbol{x}\rangle \geq \Omega_{n}-2 d M^{2} k_{n}\right)+\mathrm{P}\left(T_{\boldsymbol{W}} \geq M^{2} k_{n}\right),
$$

where the supremum over $\boldsymbol{x}$ runs over the centers of the small balls. Inserting (3.9), choosing $R=k_{n}$, and using $d \leq 1 / R$, this becomes

$$
P_{\max }\left(\Omega_{n}\right) \leq f\left(k_{n}, k_{n}\right) k_{n}^{k_{n}} \sup _{\boldsymbol{x}} \mathrm{P}\left(\langle\boldsymbol{x}, \boldsymbol{W} \boldsymbol{x}\rangle \geq \Omega_{n}-2 M^{2}\right)+\mathrm{P}\left(T_{\boldsymbol{W}} \geq M^{2} k_{n}\right) .
$$

Using Proposition 3.1, we find that

$$
\begin{aligned}
P_{\max }\left(\Omega_{n}\right) \leq & f\left(k_{n}, k_{n}\right) k_{n}^{k_{n}} \exp \left\{-\frac{1}{2} n\left(\frac{\Omega_{n}}{M^{2}}-3-\log \left(\frac{\Omega_{n}}{M^{2}}-2\right)\right)\right\}+\exp \left\{-n k_{n} I_{C_{11}^{2}}\left(M^{2}\right)\right\} \\
= & f\left(k_{n}, k_{n}\right) \exp \left\{k_{n} \log k_{n}-\frac{1}{2} n\left(\frac{\Omega_{n}}{M^{2}}-3-\log \left(\frac{\Omega_{n}}{M^{2}}-2\right)\right)\right\} \\
& +\exp \left\{-n k_{n} I_{C_{11}^{2}}\left(M^{2}\right)\right\} .
\end{aligned}
$$

We choose $\Omega_{n}=K \log n$ with $K$ so large that

$$
\frac{k_{n} \log k_{n}}{n}<\frac{1}{4}\left(\frac{\Omega_{n}}{M^{2}}-3-\log \left(\frac{\Omega_{n}}{M^{2}}-2\right)\right) \text {. }
$$

Therefore, also using the fact that

$$
f\left(k_{n}, k_{n}\right)=\mathrm{e}^{o(n \log n)},
$$

we obtain

$$
P_{\max }\left(\Omega_{n}\right) \leq \exp \left\{-\frac{K}{4 M^{2}} n \log n(1+o(1))\right\}+\exp \left\{-n k_{n} I_{C_{11}^{2}}\left(M^{2}\right)\right\} .
$$

Next, we investigate the first term of (3.10). In this term we can use $\Omega_{n}$ as the upper bound for $\lambda_{\max }$. Therefore, again starting with (2.16), we obtain, for any $R$,

$$
-\frac{1}{n} \log \mathrm{P}\left(\lambda_{\min } \leq \alpha, \lambda_{\max } \leq \Omega_{n}\right) \geq-\frac{1}{n}\left[\log M_{R}+\sup _{\boldsymbol{x}} \log \mathrm{P}\left(\langle\boldsymbol{x}, \boldsymbol{W} \boldsymbol{x}\rangle \leq \alpha+\frac{2 \Omega_{n}}{R}\right)\right] .
$$

For $\lambda_{\max }$, we get a similar expression. Inserting (3.9), we need to choose $R$ again. This time we wish to choose $R=R_{n}$ to increase in such a way that $k_{n} \log R_{n}=o(n)$ and $\Omega_{n}=K \log n=$ $o\left(R_{n}\right)$. For the latter, we need $R_{n} \gg \log n$, so that we can satisfy only the first restriction when $k_{n}=o(n / \log \log n)$. Then this is sufficient to make the term $2 \Omega_{n} / R_{n}$ disappear as $k_{n} \rightarrow \infty$, and to make the term $(1 / n)\left(\log M_{R_{n}}\right)=\mathcal{O}\left(k_{n} \log R_{n}\right)$ be $o(n)$, so that it also disappears. Therefore, for any $R=R_{n}$ satisfying the above two restrictions,

$$
-\frac{1}{n} \log \mathrm{P}\left(\lambda_{\min } \leq \alpha, \lambda_{\max } \leq \Omega_{n}\right) \geq I_{k_{n}}\left(\alpha+2 \frac{\Omega_{n}}{R_{n}}\right)+o(1) .
$$


Similarly,

$$
-\frac{1}{n} \log \mathrm{P}\left(\alpha \leq \lambda_{\max } \leq \Omega_{n}\right) \geq I_{k_{n}}\left(\alpha-2 \frac{\Omega_{n}}{R_{n}}\right)+o(1) .
$$

Moreover, by the fact that $\Omega_{n}=K \log n=o\left(R_{n}\right)$, we have $2 \Omega_{n} / R_{n} \leq \varepsilon$ for all large enough $n$. By the monotonicity of $\alpha \mapsto I_{k_{n}}(\alpha)$ we then have

$$
I_{k_{n}}\left(\alpha+\frac{2 \Omega_{n}}{R_{n}}\right) \geq I_{k_{n}}(\alpha+\varepsilon), \quad I_{k_{n}}\left(\alpha-\frac{2 \Omega_{n}}{R_{n}}\right) \geq I_{k_{n}}(\alpha-\varepsilon) .
$$

Since $\lim _{k \rightarrow \infty} I_{k}(\alpha)=I_{\infty}(\alpha)$ (see (3.8)), putting (3.13), (3.14), and (3.11) together and applying the largest-exponent-wins principle, (2.11), we see that the proof follows when

$$
I_{\infty}(\alpha \pm \varepsilon)<\min \left\{\log n \frac{K}{4 M^{2}}, k_{n} I_{C_{11}^{2}}\left(M^{2}\right)\right\} .
$$

Both terms are increasing in $n$, as long as $I_{C_{11}^{2}}\left(M^{2}\right)>0$. This is true for the $C_{11}$ we consider: if $C_{11}$ is symmetric around 0 such that $\left|C_{11}\right|^{11}<M<\infty$ a.s., then $I_{C_{11}^{2}}\left(M^{2}\right)=\infty$, and if $C_{m i}$ is standard normal then $I_{C_{11}^{2}}\left(M^{2}\right)>0$.

Therefore, (3.15) is true when $n$ is large enough, when $I_{\infty}(\alpha \pm \varepsilon)<\infty$. On the other hand, when $I_{\infty}(\alpha \pm \varepsilon)=\infty$, then we find that the exponential rates converge to $\infty$, as stated in (3.3) and (3.5). We conclude that, for every $\varepsilon>0$,

$$
\liminf _{n \rightarrow \infty}-\frac{1}{n} \log \mathrm{P}_{k_{n}}\left(\lambda_{\min } \leq \alpha\right) \geq I_{\infty}(\alpha+\varepsilon)
$$

and

$$
\liminf _{n \rightarrow \infty}-\frac{1}{n} \log \mathrm{P}_{k_{n}}\left(\lambda_{\max } \geq \alpha\right) \geq I_{\infty}(\alpha-\varepsilon),
$$

and letting $\varepsilon \downarrow 0$ completes the proof for every sequence $k_{n}$ such that $k_{n}=o(n / \log \log n)$. The proof for $\lambda_{\max }$ is identical to the above proof.

We believe that the above argument can be extended somewhat further, by making a further split into $K^{\prime} \log \log n \leq \lambda_{\max } \leq K \log n$ and $\lambda_{\max } \leq K^{\prime} \log \log n$, but we refrain from writing this down.

\subsection{The limiting rate for large $k$}

In this subsection we investigate what happens when we take $k$ large. In certain cases we can show that the rate function, which depends on $k$, converges to the rate function for Wishart matrices. This will be formulated in the following theorem.

Theorem 3.2. Assume that $C_{i j}$ satisfies (2.1) and, moreover, that $\phi_{C}(t)=\mathrm{E}\left[\exp \left\{t C_{11}\right\}\right] \leq$ $\exp \left\{t^{2} / 2\right\}$ for all $t$. Then, for all $\alpha \geq 1$ and all $k \geq 2$,

$$
I_{k}(\alpha) \geq \frac{1}{2}(\alpha-1-\log \alpha)
$$

and, for all $\alpha \geq 1$,

$$
\lim _{k \rightarrow \infty} I_{k}(\alpha)=I_{\infty}(\alpha)=\frac{1}{2}(\alpha-1-\log \alpha)
$$

Finally, for all $k_{n} \rightarrow \infty$ such that $k_{n}=o(n / \log \log n)$ and $\alpha \geq 1$,

$$
\lim _{n \rightarrow \infty}-\frac{1}{n} \log \mathrm{P}_{k_{n}}\left(\lambda_{\max } \geq \alpha\right)=\frac{1}{2}(\alpha-1-\log \alpha) .
$$


Note that, in particular, Theorem 3.2 implies that $I_{\infty}(\alpha)=\frac{1}{2}(\alpha-1-\log \alpha)>0$ for all $\alpha>1$.

Theorem 3.2 is a kind of universality result, and shows that, for large $k$, the rate functions of certain sample covariance matrices converge to the rate functions for Wishart matrices. An example where $\phi_{C}(t) \leq \exp \left\{t^{2} / 2\right\}$ holds is when $C_{i j}= \pm 1$ with equal probability. We will call this example the Bernoulli case. A second example is the uniform random variable on $[-\sqrt{3}, \sqrt{3}]$, for which the variance also equals 1 . We will prove these bounds below.

Of course, the relation $\phi_{C}(t) \leq \exp \left\{t^{2} / 2\right\}$ for random variables with mean 0 and variance 1 is equivalent to the statement that $\phi_{C}(t) \leq \exp \left\{t^{2} \sigma^{2} / 2\right\}$ for a random variable $C$ with mean 0 and variance $\sigma^{2}$. Thus, we will check the condition for uniform random variables on $[-1,1]$ and for the Bernoulli case. We will denote the moment generating functions by $\phi_{U}$ and $\phi_{B}$. We start with $\phi_{B}$, for which we have

$$
\phi_{B}(t)=\cosh (t)=\sum_{n=0}^{\infty} \frac{t^{2 n}}{(2 n) !} \leq \sum_{n=0}^{\infty} \frac{t^{2 n}}{2^{n} n !}=\exp \left\{\frac{t^{2}}{2}\right\},
$$

since $(2 n) ! \geq 2^{n} n$ ! for all $n \geq 0$. The proof for $\phi_{U}$ is similar. Indeed,

$$
\phi_{U}(t)=\frac{\sinh (t)}{t}=\sum_{n=0}^{\infty} \frac{t^{2 n}}{(2 n+1) !} \leq \sum_{n=0}^{\infty} \frac{t^{2 n}}{6^{n} n !}=\exp \left\{\frac{t^{2}}{6}\right\}=\exp \left\{\frac{t^{2} \sigma^{2}}{2}\right\},
$$

since now $(2 n+1) ! \geq 6^{n} n$ ! for all $n \geq 0$.

Proof of Theorem 3.2. Using Theorems 2.1 and 3.1, we claim that it suffices to prove that, uniformly for $\boldsymbol{x}$ with $\|\boldsymbol{x}\|_{2}=1$ and $t<1$,

$$
\mathrm{E}\left[\exp \left\{t S_{\boldsymbol{x}, i}^{2}\right\}\right] \leq \frac{1}{\sqrt{1-2 t}} .
$$

We will prove (3.19) below, but first we prove Theorem 3.2, assuming that (3.19) holds.

When (3.19) holds, then, using Theorem 3.1 and (2.4), it immediately follows that (3.16) holds. Here we also use the fact that $\alpha \mapsto \frac{1}{2}(\alpha-1-\log \alpha)$ is continuous, so that the limit over $\varepsilon \downarrow 0$ can be computed.

To prove (3.17), we take $\boldsymbol{x}=(1 / \sqrt{k})(1, \ldots, 1)$, to obtain, with $S_{k}=\sum_{i=1}^{k} C_{i 1}$,

$$
I_{k}(\alpha) \leq \sup _{t}\left(t \alpha-\log \mathrm{E}\left[\exp \left\{\frac{t}{k} S_{k}^{2}\right\}\right]\right) .
$$

We claim that, when $k \rightarrow \infty$, for all $0 \leq t<1$,

$$
\mathrm{E}\left[\exp \left\{\frac{t}{k} S_{k}^{2}\right\}\right] \rightarrow \mathrm{E}\left[\exp \left\{t Z^{2}\right\}\right]=\frac{1}{\sqrt{1-2 t}},
$$

where $Z$ is a standard normal random variable. This implies the lower bound for $I_{k}(\alpha)$ and, thus, (3.17). Equation (3.18) follows in a similar way, also using the fact that $\alpha \mapsto \frac{1}{2}(\alpha-1-\log \alpha)$ is continuous.

We complete the proof by showing that (3.19) and (3.20) hold. We start with (3.19). We rewrite, for $t \geq 0$,

$$
\mathrm{E}\left[\exp \left\{t S_{\boldsymbol{x}, i}^{2}\right\}\right]=\mathrm{E}\left[\exp \left\{\sqrt{2 t} Z S_{\boldsymbol{x}, i}\right\}\right]=\mathrm{E}\left[\prod_{j=1}^{k} \phi_{C}\left(\sqrt{2 t} Z \boldsymbol{x}_{j}\right)\right],
$$


where $Z$ is a standard normal random variable. We now use the fact that $\phi_{C}(t) \leq \exp \left\{t^{2} / 2\right\}$ to arrive at

$$
\mathrm{E}\left[\exp \left\{t S_{x, i}^{2}\right\}\right] \leq \mathrm{E}\left[\prod_{j=1}^{k} \exp \left\{t Z^{2} x_{j}^{2}\right\}\right]=\mathrm{E}\left[\exp \left\{t Z^{2}\right\}\right]=\frac{1}{\sqrt{1-2 t}} .
$$

This completes the proof of (3.19). We proceed with (3.20). We use

$$
\mathrm{E}\left[\exp \left\{t S_{k}^{2}\right\}\right]=\mathrm{E}\left[\prod_{j=1}^{k} \phi_{C}\left(\sqrt{\frac{2 t}{k}} Z\right)\right] .
$$

We will use dominated convergence. By the assumption that $\phi_{C}(t) \leq \exp \left\{t^{2} / 2\right\}$, we have

$$
\phi_{C}\left(\sqrt{\frac{2 t}{k}} Z\right) \leq \exp \left\{\frac{t}{k} Z^{2}\right\}, \quad \text { so that } \prod_{j=1}^{k} \phi_{C}\left(\sqrt{\frac{2 t}{k}} Z\right) \leq \exp \left\{t Z^{2}\right\},
$$

which has a finite expectation when $t<\frac{1}{2}$. Moreover, $\prod_{j=1}^{k} \phi_{C}\left((2 t / k)^{1 / 2} z\right)$ converges to $\exp \left\{t z^{2}\right\}$ pointwise in $z$. Therefore, dominated convergence proves the claim in (3.20), and completes the proof.

\section{The smallest eigenvalue for $C_{i j}= \pm 1$}

Unfortunately, we are not able to prove a similar result as in Theorem 3.2 for the smallest eigenvalue. In fact, as we will comment on in more detail in Subsection 4.2, we expect the result to be false for the smallest eigenvalue, in particular, when $\alpha$ is small. There is one example where we can prove a partial convergence result, and that is when $C_{i j}= \pm 1$ with equal probability. Indeed, in this case it was shown in [18, Section IV] that (3.19) holds for all $t \geq-1$. This leads to the following result, which also implies that $I_{k}(\alpha)>0$ for $\alpha \neq 1$ in the case where $\operatorname{var}\left(C_{11}^{2}\right)=0$ (recall also Theorem 2.1).

Theorem 4.1. Assume that $C_{i j}= \pm 1$ with equal probability. Then, for all $\alpha \geq \frac{1}{2}$ and all $k \geq 2$,

$$
I_{k}(\alpha) \geq \frac{1}{2}(\alpha-1-\log \alpha),
$$

and, for all $\alpha \geq \frac{1}{2}$,

$$
I_{\infty}(\alpha)=\frac{1}{2}(\alpha-1-\log \alpha)
$$

Finally, for all $0<\alpha \leq \frac{1}{2}$,

$$
I_{k}(\alpha) \geq \frac{1}{2}(-\alpha+\log 2) .
$$

Proof. The proofs of (4.1) and (4.2) are identical to the proof of Theorem 3.2, now assuming that (3.19) holds for all $t \geq-1$. Equation (4.3) follows since

$$
I_{k}(\alpha) \geq \inf _{x:\|x\|_{2}=1}\left(-\frac{\alpha}{2}-\log \mathrm{E}\left[\exp \left\{-\frac{1}{2} S_{x, i}^{2}\right\}\right]\right)
$$

and the bound on the moment generating function in (3.19), which, as discussed above, holds for all $t \geq-1$ when $C_{i j}= \pm 1$ with equal probability, for $t=-1$. 


\subsection{Rate for the probability of one or more zero eigenvalues for $\boldsymbol{C}_{\boldsymbol{i j}}= \pm \mathbf{1}$}

In the above computations we obtain no control over the probability of a large deviation of the smallest eigenvalue, $\lambda_{\min }$. In this and the next subsection we investigate this problem for the case in which $C_{i j}= \pm 1$.

Proposition 4.1. Suppose that $C_{i j}= \pm 1$ with equal probability. Then, for all $0<l \leq k-1$ and any $k_{n}=\mathcal{O}\left(n^{b}\right)$ for some $b$,

$$
\lim _{n \rightarrow \infty}-\frac{1}{n} \log \mathrm{P}_{k_{n}}\left(\lambda_{1}=\cdots=\lambda_{l}=0\right)=l \log 2,
$$

where the $\lambda_{i}$ denote eigenvalues of $\boldsymbol{W}$ arranged in increasing order.

Proof. The upper bound in (4.4) is simple, since, to have $l$ eigenvalues equal to 0 , we can take the first $l+1$ columns of $\boldsymbol{C}$ to be equal. For eigenvectors $\boldsymbol{w}$ of $\boldsymbol{W}$,

$$
\langle\boldsymbol{w}, \boldsymbol{W} \boldsymbol{w}\rangle=\frac{1}{n}\left\langle\boldsymbol{w}, \boldsymbol{C} \boldsymbol{C}^{\top} \boldsymbol{w}\right\rangle=\frac{1}{n}\left\|\boldsymbol{C}^{\top} \boldsymbol{w}\right\|_{2}^{2},
$$

we find that $\boldsymbol{w}$ is an eigenvector with eigenvalue 0 precisely when $\left\|\boldsymbol{C}^{\top} \boldsymbol{w}\right\|_{2}=0$. When the first $l+1$ columns of $\boldsymbol{C}$ are equal, there are $l$ linearly independent vectors for which $\left\|\boldsymbol{C}^{\top} \boldsymbol{w}\right\|_{2}=0$, so that the multiplicity of the eigenvalue 0 is at least $l$. Moreover, the probability that the first $l+1$ columns of $\boldsymbol{C}$ are equal is equal to $2^{n l}$.

We prove the lower bound in (4.4) by induction by showing that

$$
\lim _{n \rightarrow \infty}-\frac{1}{n} \log \mathrm{P}_{k_{n}}\left(\lambda_{1}=\cdots=\lambda_{l}=0\right) \geq l \log 2 .
$$

When $l=0$, the claim is trivial. It suffices to advance the induction hypothesis.

Suppose that there are $l$ linear independent eigenvectors with eigenvalue 0 . Since the eigenvectors can be chosen to be orthogonal, it is possible to make linear combinations, such that the first $l-1$ eigenvectors all have one zero coordinate $j$, whereas the $l$ th eigenvectors has all zero coordinates except for coordinate $j$. This means that the first $l-1$ eigenvectors fix some part of $\boldsymbol{C}^{\top}$, but not the $j$ th column. The $l$ th eigenvector however fixes precisely this column. Fixing one column of $\boldsymbol{C}^{\top}$ has probability $2^{-n}$. The number of possible rows $j$ is bounded by $k$, which in turn is bounded by $n^{b}=\mathrm{e}^{o(n)}$. Therefore, we have

$$
\begin{aligned}
\lim _{n \rightarrow \infty} & -\frac{1}{n} \log \mathrm{P}_{k_{n}}\left(\lambda_{1}=\cdots=\lambda_{l}=0\right) \\
& \geq \log 2+\lim _{n \rightarrow \infty}-\frac{1}{n} \log \mathrm{P}_{k_{n}}\left(\lambda_{1}=\cdots=\lambda_{l-1}=0\right) .
\end{aligned}
$$

The claim follows from the induction hypothesis.

Note that Proposition 4.1 shows that (4.2) cannot be extended to $\alpha=0$. Therefore, a changeover takes place between $\alpha=0$ and $\alpha \geq \frac{1}{2}$, where, for $\alpha \geq \frac{1}{2}$, the rate function equals the one for Wishart matrices, while, for $\alpha=0$, this is not the case. We will comment more on this in Conjecture 4.1, below.

\subsection{A conjecture about the smallest eigenvalue for $C_{i j}= \pm 1$}

We have already shown that (4.1) is sharp. By Proposition 4.1, (4.3) is not sharp, since $I_{\min }(0)=\log 2$, whereas (4.3) only yields $\lim _{\alpha \downarrow 0} I_{\min }(\alpha) \geq \frac{1}{2} \log 2$. 
We can use (2.15) again with $\boldsymbol{x}^{\prime}=(1 / \sqrt{2})(1,1,0, \ldots)$. For this vector, $\mathrm{E}\left[\exp \left\{t S_{\boldsymbol{x}^{\prime}, i}^{2}\right\}\right]=$ $\frac{1}{2}\left(\mathrm{e}^{2 t}+1\right)$, and calculating the corresponding rate function gives

$$
I_{k}(\alpha) \leq I^{(2)}(\alpha)=\frac{\alpha}{2} \log \alpha+\frac{2-\alpha}{2} \log (2-\alpha),
$$

which implies that $\lim _{\alpha \downarrow 0} I_{k}(\alpha) \leq \log 2$.

It appears that below a certain $\alpha=\alpha_{k}^{*}$, the optimal strategy changes from

$$
\boldsymbol{x}^{(k)}=\frac{1}{\sqrt{k}}(1,1, \ldots) \quad \text { to } \quad \boldsymbol{x}^{(2)}=\frac{1}{\sqrt{2}}(1,1,0, \ldots) .
$$

In words, this means that, for not too small eigenvalues of $\boldsymbol{W}$, all entries of $\boldsymbol{C}$ contribute equally to create a small eigenvalue. However, smaller values of the smallest eigenvalues of $\boldsymbol{W}$ are created by only two columns of $\boldsymbol{C}$, whereas the others are close to orthogonal. Thus, a change in strategy occurs, which gives rise to a phase transition in the asymptotic exponential rate for the smallest eigenvalue.

We have the following conjecture.

Conjecture 4.1. For each $k$ and all $\alpha \geq 0$, there exists an $\alpha^{*}=\alpha_{k}^{*}>0$ such that

$$
I_{k}(\alpha)=I^{(2)}(\alpha)=\frac{\alpha}{2} \log \alpha+\frac{(2-\alpha)}{2} \log (2-\alpha) \quad \text { for } \alpha \leq \alpha_{k}^{*} .
$$

For $\alpha>\alpha_{k}^{*}$

$$
I^{(2)}(\alpha)>I_{k}(\alpha) \geq I_{\infty}(\alpha)=\frac{1}{2}(\alpha-1-\log \alpha) .
$$

For $k \rightarrow \infty$, the last inequality will become an equality. Consequently, $\lim _{k \rightarrow \infty} \alpha_{k}^{*}=\alpha^{*}$, which is the positive solution of $I_{\infty}(\alpha)=I^{(2)}(\alpha)$.

For $k=2$, the conjecture is trivially true, since the two optimal strategies are the same, and the only possible. Note that in the proof of Proposition 4.1 we needed two vectors of $C$ to be equal in order to have a zero eigenvalue. Thus, the conjecture is also proven for $\alpha=0$. Furthermore, with Theorem 3.1 and (3.6), the conjecture follows for all $k$ for $\alpha \geq \frac{1}{2}$. We lack a proof for $0<\alpha<\frac{1}{2}$. Numerical evaluation gives $\alpha_{3}^{*} \approx 0.425$ and, for $k \rightarrow \infty, \alpha_{k}^{*} \approx 0.253$. We have some evidence that suggests that $\alpha_{k}^{*}$ decreases with $k$.

\section{An application: mobile communication systems}

Our results on the eigenvalues of sample covariance matrices were triggered by a problem in mobile communication systems. In this case we take the $\boldsymbol{C}$ matrix as a coding sequence, for which we can assume that the elements are \pm 1 . Thus, all our results apply to this case. In this section we will describe the consequences of our results on this problem.

\subsection{Soft-decision parallel interference cancellation}

In code-division multiple-access (CDMA) communication systems, each of the $k$ users multiplies his data signal by an individual coding sequence. The base station can distinguish the different messages by taking the inner product of the total signal with each coding sequence. This is called matched filter (MF) decoding. An important application is mobile telephony. Since, owing to synchronization problems, it is unfeasible to implement completely orthogonal codes for mobile users, the decoded messages will suffer from multiple-access interference (MAI). 
In practice, pseudorandom codes are used. Designers of decoding schemes are interested in the probability that a decoding error is made.

In the following we explain a specific method to iteratively estimate and subtract the MAI, namely, soft-decision parallel interference cancellation (SD-PIC). For more background on SD-PIC, see [5], [9], [11], and [13], as well as the references therein. Because this procedure is linear, it can be expressed in matrix notation. We will show that the possibility of a decoding error is related to a large deviation of the maximum or minimum eigenvalue of the code correlation matrix.

To highlight the aspects that are relevant in this paper, we suppose that each user sends only one data bit $b_{m} \in\{+1,-1\}$, and we omit noise from additional sources. We can denote all sent data multiplied by their amplitude in a column vector $\boldsymbol{Z}$, i.e. $\boldsymbol{Z}_{m}=\sqrt{P_{m}} b_{m}$, where $P_{m}$ is the power of the $m$ th user. The $k$ codes are modeled as the different rows of length $n$ of the code matrix $\boldsymbol{C}$, consisting of i.i.d. random bits with distribution

$$
\mathrm{P}\left(C_{m i}=+1\right)=\mathrm{P}\left(C_{m i}=-1\right)=\frac{1}{2} .
$$

Thus, $k$ plays the role of the number of users, while $n$ is the length of the different codes.

The base station then receives a total signal $s=\boldsymbol{C}^{\top} \boldsymbol{Z}$. Decoding for user $m$ is done by taking the inner product with the code of the $m$ th user, $\left(C_{1 m}, \ldots, C_{n m}\right)$, and dividing by $n$. This yields an estimate $\hat{\boldsymbol{Z}}_{m}^{(1)}$ for the sent signal $\boldsymbol{Z}_{m}$. In matrix notation, the vector $\boldsymbol{Z}$ is estimated by

$$
\hat{\boldsymbol{Z}}^{(1)}=\frac{1}{n} \boldsymbol{C s}=\boldsymbol{W Z} \text {. }
$$

Thus, we see that multiplying with the matrix $\boldsymbol{W}$ is equivalent to the MF decoding scheme. In order to estimate the signal, we must find the inverse matrix $\boldsymbol{W}^{-1}$. From $\hat{\boldsymbol{Z}}^{(1)}$ we estimate the sent bit $b_{m}$ by

$$
\hat{b}_{m}^{(1)}=\operatorname{sgn}\left(\hat{\boldsymbol{Z}}_{m}^{(1)}\right)
$$

(where, when $\hat{\boldsymbol{Z}}_{m}^{(1)}=0$, we toss an independent fair coin to decide what the value of $\operatorname{sgn}\left(\hat{\boldsymbol{Z}}_{m}^{(1)}\right)$ is). Below, we explain the role of the eigenvalues of $\boldsymbol{W}$ in the more advanced SD-PIC decoding scheme.

The MF estimate contains MAI. When we write $\hat{\boldsymbol{Z}}=\boldsymbol{Z}+(\boldsymbol{W}-\boldsymbol{I}) \boldsymbol{Z}$, where $\boldsymbol{I}$ is the $k$-dimensional identity matrix, it is clear that the estimated bit vector is a sum of the correct bit vector and MAI. In SD-PIC, the second term is subtracted, with $\boldsymbol{Z}$ replaced by $\hat{\boldsymbol{Z}}$. In the case of multistage PIC, each new estimate is used in the next PIC iteration. We will now write the multistage SD-PIC procedure in matrix notation. We number the successive SD estimates for $\boldsymbol{Z}$ with an index $s$, where $s=1$ corresponds to the MF decoding. In each new iteration, the latest guess for the MAI is subtracted. The iteration in a recursive form is therefore

$$
\hat{\boldsymbol{Z}}^{(s)}=\hat{\boldsymbol{Z}}^{(1)}-(\boldsymbol{W}-\boldsymbol{I}) \hat{\boldsymbol{Z}}^{(s-1)} .
$$

This can be reformulated as

$$
\hat{\boldsymbol{Z}}^{(s)}=\sum_{\varsigma=0}^{s-1}(\boldsymbol{I}-\boldsymbol{W})^{\varsigma} \boldsymbol{W} \boldsymbol{Z} .
$$

We then estimate $b_{m}$ by

$$
\hat{b}_{m}^{(s)}=\operatorname{sgn}\left(\hat{\boldsymbol{Z}}_{m}^{(s)}\right) .
$$

When $s \rightarrow \infty$, the series $\sum_{\zeta=0}^{s-1}(\boldsymbol{I}-\boldsymbol{W})^{\varsigma}$ converges to $\boldsymbol{W}^{-1}$, as long as the eigenvalues of $\boldsymbol{W}$ are between 0 and 2. Otherwise, a decoding error is made. This is the crux to the method; see 
also [16] for the above matrix computations. When $k=o(n / \log \log n)$, the values $\lambda_{\min }=0$ and $\lambda_{\max } \geq 2$ are large deviations, and, therefore, our derived rate functions provide information on the error probability. In the next subsection we will describe these results, and we will also obtain bounds on the exponential rate of a bit error in the case that $s$ is fixed and $k$ is large. For an extensive introduction to CDMA and PIC procedures, we refer the reader to [13].

\subsection{Results for SD-PIC}

There are two cases that need to be distinguished, namely, the case where $s \rightarrow \infty$ and the case where $s$ is fixed. We start with the former, which is simplest. As explained in the previous subsection, due to the absence of noise, there can only be bit errors when $\lambda_{\min }=0$ or when $\lambda_{\max } \geq 2$. By (4.3), the rate of $\lambda_{\min }=0$ is at least $\frac{1}{2} \log 2 \approx 0.35 \ldots$, whereas the rate of $\lambda_{\max } \geq 2$ is bounded below by $\frac{1}{2}-\frac{1}{2} \log 2 \approx 0.15 \ldots$. The latter bound is weaker and, thus, by the largest-exponent-wins principle, we obtain the following result.

Theorem 5.1. (Bit-error rate for optimal SD-PIC.) For all $k$ fixed, or for $k=k_{n} \rightarrow \infty$ such that $k_{n}=o(n / \log \log n)$,

$$
-\frac{1}{n} \log \mathrm{P}_{k}\left(\text { there exists } m=1, \ldots, k \text { for which } \lim _{s \rightarrow \infty} \hat{b}_{m}^{(s)} \neq b_{m}\right) \geq \frac{1}{2}-\frac{1}{2} \log 2 .
$$

We emphasize that in the statement of the result we write that $\lim _{s \rightarrow \infty} \hat{b}_{m}^{(s)} \neq b_{m}$ for all $m=1, \ldots, k$ for the statement that either $\lim _{s \rightarrow \infty} \hat{b}_{m}^{(s)}$ does not exist or that $\lim _{s \rightarrow \infty} \hat{b}_{m}^{(s)}$ exists, but is unequal to $b_{m}$. We observe that, when $\lambda_{\max }>2$,

$$
\hat{\boldsymbol{Z}}^{(s)}=\sum_{\varsigma=0}^{s-1}(\boldsymbol{I}-\boldsymbol{W})^{\varsigma} \boldsymbol{W} \boldsymbol{Z}
$$

oscillates, so that we can expect there to be errors in every stage. This is sometimes called the ping-pong effect (see [16]). Thus, we would expect that

$$
-\frac{1}{n} \log \mathrm{P}_{k_{n}}\left(\text { there exists } m=1, \ldots, k \text { for which } \lim _{s \rightarrow \infty} \hat{b}_{m}^{(s)} \neq b_{m}\right)=\frac{1}{2}-\frac{1}{2} \log 2 .
$$

However, this also depends on the relation between $\boldsymbol{Z}$ and the eigenvector corresponding to $\lambda_{\max }$. Indeed, when $\boldsymbol{Z}$ is orthogonal to the eigenvector corresponding to $\lambda_{\max }$, the equality does not follow. To avoid such problems, we stick to lower bounds on the rates in this subsection, rather than asymptotics.

Next we examine the case in which $s$ is fixed. We again consider the case where $k$ is large and fixed, or where $k=k_{n} \rightarrow \infty$. In this case, it can be expected that the rate converges to 0 as $k \rightarrow \infty$. We already know that the probability that $\lambda_{\max } \geq 2$ or $\lambda_{\min }=0$ is exponentially small with fixed strictly positive lower bound on the exponential rate. Thus, we will assume that $0<\lambda_{\min } \leq \lambda_{\max }<2$. We can then rewrite

$$
\hat{\boldsymbol{Z}}^{(s)}=\sum_{s=0}^{s-1}(\boldsymbol{I}-\boldsymbol{W})^{\varsigma} \boldsymbol{W} \boldsymbol{Z}=\left[\boldsymbol{I}-(\boldsymbol{I}-\boldsymbol{W})^{s}\right] \boldsymbol{Z} .
$$

For simplicity, we will first assume that $Z_{i}= \pm 1$ for all $i=1, \ldots, k$, which is equivalent to assuming that all powers are equal. When $s$ is fixed, we cannot have any bit errors when

$$
\left|\left((\boldsymbol{I}-\boldsymbol{W})^{s} \boldsymbol{Z}\right)_{i}\right|<1 .
$$


We can bound

$$
\left|\left((\boldsymbol{I}-\boldsymbol{W})^{s} \boldsymbol{Z}\right)_{i}\right| \leq \varepsilon_{k}^{s}\|\mathbf{b}\|_{2},
$$

where $\varepsilon_{k}=\max \left\{1-\lambda \lambda_{\min }, \lambda_{\max }-1\right\}$. Since $\|\mathbf{b}\|_{2}=\sqrt{k}$, there cannot be any bit errors when $\varepsilon_{k}^{s} \sqrt{k}<1$. This gives an explicit relation between the bit errors and the eigenvalues of a random sample covariance matrix. By applying the results from Sections 3 and 4, we obtain the following theorem.

Theorem 5.2. (Bit-error rate for finite-stage SD-PIC and fixed $k$.) For all $k$ such that $k>2^{2 s}$,

$$
\begin{aligned}
& -\liminf _{n \rightarrow \infty} \frac{1}{n} \log \mathrm{P}_{k}\left(\text { there exists } m=1, \ldots, k \text { for which } \lim _{s \rightarrow \infty} \hat{b}_{m}^{(s)} \neq b_{m}\right) \\
& \geq \frac{1}{4 \sqrt[s]{k}}\left(1+\mathcal{O}\left(\frac{1}{\sqrt[s]{k}}\right)\right) .
\end{aligned}
$$

When the signals are different, related results can be obtain in terms of the minimal and maximal elements of $\boldsymbol{Z}$. We will not write this case out.

Proof of Theorem 5.2. By the computation in (5.2), there can be no bit errors when $1-\lambda_{\min }$ and $\lambda_{\max }-1$ are both at most $1 / \sqrt[2 s]{k}$. Thus,

$$
\begin{array}{r}
\mathrm{P}_{k}\left(\text { there exists } m=1, \ldots, k \text { for which } \lim _{s \rightarrow \infty} \hat{b}_{m}^{(s)} \neq b_{m}\right) \\
\leq \mathrm{P}_{k}\left(\lambda_{\min } \leq 1-\frac{1}{\sqrt[2 s]{k}}\right)+\mathrm{P}_{k}\left(\lambda_{\max } \geq 1+\frac{1}{\sqrt[2 s]{k}}\right)
\end{array}
$$

Each of these terms is bounded by, using Theorem 2.1,

$$
\exp \left\{-n \min \left\{\lim _{\varepsilon \downarrow 0} I_{k}\left(1-\frac{2}{\sqrt[2 s]{k}}-\varepsilon\right), I_{k}\left(1+\frac{2}{\sqrt[2 s]{k}}+\varepsilon\right)\right\}(1+o(1))\right\} .
$$

Since, by Theorem 4.1 and $\alpha \geq \frac{1}{2}$, we have $I_{k}(\alpha) \geq I_{\infty}(\alpha)=\frac{1}{2}(\alpha-1-\log \alpha)$, and

$$
I_{\infty}(\alpha)=\frac{1}{4}(\alpha-1)^{2}+\mathcal{O}\left(|\alpha-1|^{3}\right),
$$

the result follows when $k$ is so large that $1-1 / \sqrt[2 s]{k}>\frac{1}{2}$. The latter is equivalent to $k>2^{2 s}$.

We finally state a result that applies to $k=k_{n}$.

Theorem 5.3. (Bit-error rate for finite-stage SD-PIC and $k=k_{n}$.) For $k_{n}=o\left(n^{s /(s+1)} / \log n\right)$,

$$
-\frac{\sqrt[s]{k_{n}}}{n} \log \mathrm{P}_{k_{n}}\left(\text { there exists } m=1, \ldots, k \text { for which } \lim _{s \rightarrow \infty} \hat{b}_{m}^{(s)} \neq b_{m}\right) \geq \frac{1}{4}+\mathcal{O}\left(\frac{1}{\sqrt[s]{k_{n}}}\right) \text {. }
$$

Proof. We use (5.3) to conclude that we need to derive bounds for $\mathrm{P}_{k_{n}}\left(\lambda_{\min } \leq 1-1 / \sqrt[2 s]{k_{n}}\right)$ and $\mathrm{P}_{k_{n}}\left(\lambda_{\max } \geq 1+1 / \sqrt[2 s]{k_{n}}\right)$. Unfortunately, the bounds $1-1 / \sqrt[2 s]{k_{n}}$ and $1+1 / \sqrt[2 s]{k_{n}}$ on the smallest and largest eigenvalues depend on $n$, rather than being fixed. Therefore, we need to adapt the proof of Theorem 3.1.

We note that, by Theorem 3.1,

$$
\mathrm{P}_{k_{n}}\left(\lambda_{\max } \geq 2\right)=\exp \left\{-\left(\frac{1}{2}-\frac{1}{2} \log 2\right) n(1+o(1))\right\} .
$$


Then, we use (3.13) with $\Omega_{n}=2$, and choose $R_{n}$ such that

$$
\frac{2}{R_{n}}=o\left(\frac{1}{\sqrt[2 s]{k_{n}}}\right)
$$

so that $R_{n} \gg \sqrt[2 s]{k_{n}}$. Applying (3.12) and (3.9), we see that we need $R_{n}^{k_{n}}=\exp \left\{o\left(n / \sqrt[s]{k_{n}}\right)\right\}$, so that $k_{n}=o\left(n^{s /(s+1)} / \log n\right)$ is sufficient. Finally, by Theorem 4.1 and (5.4), we have

$$
I_{k_{n}}\left(1 \pm \frac{1}{\sqrt[2 s]{k_{n}}}\right) \geq \frac{1}{4 \sqrt[s]{k_{n}}}\left(1+\mathcal{O}\left(\frac{1}{\sqrt[s]{k_{n}}}\right)\right)
$$

This completes the proof.

We now discuss the above results. In [20], it was conjectured that, when $s=2$, the rate of a single bit error for a fixed user is asymptotic to $1 / 2 \sqrt{k}$ when $k \rightarrow \infty$. See also [21]. We see that we obtain a similar result, but our constant is $\frac{1}{4}$ rather than the expected $\frac{1}{2}$. On the other hand, our result is valid for all $s \geq 2$.

Related results where obtained for a related model, hard-decision parallel interference cancellation (HD-PIC), where bits are iteratively estimated by bits, i.e. the estimates are rounded to \pm 1 . Thus, this scheme is not linear, as SD-PIC is. In [18] and [19], similar results to the above were obtained, and it was shown that the rate for a bit error for a given user is asymptotic to $(s / 8) \sqrt[s]{4 / k}$ when $s$ is fixed and $k \rightarrow \infty$. This result is similar in spirit to the one in Theorem 5.2. The explanation of why the rate tends to 0 as $1 / \sqrt[s]{k}$ is much simpler for the case of SD-PIC, where the relation to eigenvalues is rather direct, compared to the explanation for HD-PIC, which is much more elaborate. It is interesting to see that both when $s=\infty$ and when $s$ is finite and $k \rightarrow \infty$, the rates in the two systems are of the same order.

Interestingly, in [22], it was shown that, for $s=1$ and $k_{n}=n / \gamma \log n$, with high probability, all bits are estimated correctly when $\gamma<2$, while, with high probability, there is at least one bit error when $\gamma>2$. Thus, $k_{n}=\mathcal{O}(n / \log n)$ is critical for the MF system, where we do not apply SD-PIC. For SD-PIC with an arbitrary number of stages of SD-PIC, we have no bit errors with large probability for all $k_{n}=n / \gamma \log n$ for all $\gamma>0$, and we can even pick larger values of $k_{n}$ such that $k_{n}=o(n / \log \log n)$. Thus, SD-PIC is more efficient than MF, in the sense that it allows more users to transmit without creating bit errors. Furthermore, in [22], the results proved in this paper are used for a further comparison between SD-PIC, HD-PIC, and MF. Unfortunately, when we apply only a finite number of stages of SD-PIC, we can allow only for $k_{n}=o\left(n^{s /(s+1)} / \log n\right)$ users. Similar results were obtained for HD-PIC when $k_{n}=o(n / \log n)$.

We close this discussion on SD-PIC and HD-PIC by noting that, for $k=\beta n, \lambda_{\min }$ converges to $(1-\sqrt{\beta})_{+}^{2}$, while the largest eigenvalue, $\lambda_{\max }$, converges to $(1+\sqrt{\beta})^{2}$ (see [3], [4], and [23]). This is explained in more detail in [9], and illustrates that SD-PIC has no bit errors with probability converging to 1 whenever $\beta<(\sqrt{2}-1)^{2} \approx 0.17 \ldots$ However, unlike the case where $k_{n}=o(n / \log \log n)$, we do not obtain bounds on how the probability of a bit error tends to 0 .

A further CDMA system is the decorrelator, which explicitly inverts the matrix $\boldsymbol{W}$ (without approximating it by the partial sum $\left.\sum_{S=0}^{s-1}(\boldsymbol{I}-\boldsymbol{W})^{\varsigma}\right)$. One way of doing so is to fix a large value $M$ and to compute

$$
\hat{\boldsymbol{Z}}_{M}^{(s)}=M^{-1} \sum_{\varsigma=0}^{s-1}\left(\boldsymbol{I}-\frac{\boldsymbol{W}}{M}\right)^{\varsigma} \boldsymbol{W} \boldsymbol{Z}
$$

and

$$
\hat{b}_{m, M}^{(s)}=\operatorname{sgn}\left(\hat{\boldsymbol{Z}}_{m, M}^{(s)}\right) .
$$


This is a certain weighted SD-PIC scheme. This scheme will converge to $\boldsymbol{b}$ as $s \rightarrow \infty$ whenever $\lambda_{\min }>0$ and $\lambda_{\max }<M$. By taking $M$ such that $I_{\infty}(M) \geq \log 2$, and using Proposition 4.1, we obtain the following result.

Theorem 5.4. (Bit-error rate for optimal weighted SD-PIC.) For all $k$ fixed, or for $k=k_{n} \rightarrow \infty$ such that $k_{n}=o(n / \log \log n)$ and $M$ such that $I_{\infty}(M) \geq \log 2$,

$$
-\frac{1}{n} \log \mathrm{P}_{k}\left(\text { there exists } m=1, \ldots, k \text { for which } \hat{b}_{m, M}^{(s)} \neq b_{m}\right) \geq \log 2 \text {. }
$$

The above result can even be generalized to $k_{n}$ that grow arbitrarily fast with $n$, by taking $M$ dependent on $n$. For example, when we take $M>k_{n}, \lambda_{\max } \leq k_{n}<M$ is guaranteed.

Further interesting problems arise when we allow the received signal to be noisy. In this case, the bit error can be caused either by the properties of the eigenvalues, as in the case when there is no noise, or by the noise. When there is noise, weighted SD-PIC for large $M$ enhances the noise, which makes the problem significantly harder. See [13] for further details. A solution to Conjecture 4.1 may prove to be useful in such an analysis.

\section{Acknowledgements}

The work of AF and RvdH was supported in part by NWO. The work of RvdH and MJK was performed in part at Delft University of Technology.

\section{References}

[1] Bai, Z. D. AND Silverstein, J. W. (1998). No eigenvalues outside the support of the limiting spectral distribution of large-dimensional sample covariance matrices. Ann. Prob. 26, 316-345.

[2] Bai, Z. D. And Silverstein, J. W. (2004). CLT for linear spectral statistics of large-dimensional sample covariance matrices. Ann. Prob. 32, 553-605.

[3] BAI, Z. D. AND YIN, Y. Q. (1993). Limit of the smallest eigenvalue of a large-dimensional sample covariance matrix. Ann. Prob. 21, 1275-1294.

[4] Bai, Z. D., Silverstein, J. W. And Yin, Y. Q. (1988). A note on the largest eigenvalue of a large-dimensional sample covariance matrix. J. Multivariate Anal. 26, 166-168.

[5] Buehrer, R. M., Nicoloso, S. P. And Gollamudi, S. (1999). Linear versus non-linear interference cancellation. J. Commun. Networks 1, 118-133.

[6] Dembo, A. And Zeitouni, O. (1998). Large Deviations Techniques and Applications, 2nd edn. Springer, New York.

[7] Den Hollander, F. (2000). Large Deviations (Fields Inst. Monogr. 14). American Mathematical Society, Providence, RI.

[8] Fey-den Boer, A. C., van der Hofstad, R. and Klok, M. J. (2003). Linear interference cancellation in CDMA systems and large deviations of the correlation matrix eigenvalues. In Proc. Symp. IEEE Benelux Chapter Commum. Veh. Tech. (Eindhoven, 2003).

[9] Grant, A. And Schlegel, C. (2001). Convergence of linear interference cancellation multiuser receivers. IEEE Trans. Commun. 49, 1824-1834.

[10] Guionnet, A. (2002). Large deviations asymptotics for spherical integrals. J. Funct. Anal. 188, 461-515.

[11] Guo, D., Rasmussen, L. K., Tun, S. and Lim, T. J. (2000). A matrix-algebraic approach to linear parallel interference cancellation in CDMA. IEEE Trans. Commun. 48, 152-161.

[12] Hiai, F. And Petz, D. (1998). Eigenvalue density of the Wishart matrix and large deviations. Infin. Dimens. Anal. Quantum Prob. 1, 633-646.

[13] KLOK, M. J. (2001). Performance analysis of advanced third generation receivers. Doctoral Thesis, Delft University of Technology.

[14] Marchenko, V. A. And Pastur, L. A. (1967). The distribution of eigenvalues in certain sets of random matrices. Mat. Sb. 72, 507-536.

[15] Olivieri, E. and Vares, M. E. (2005). Large Deviations and Metastability (Encyclopedia Math. Appl. 100). Cambridge University Press.

[16] Rasmussen, L. K. ANd Oppermann, I. J. (2003). Ping-pong effects in linear parallel interference cancellation for CDMA. IEEE Trans. Wireless Commun. 2, 357-363. 
[17] Rogers, C. A. (1963). Covering a sphere with spheres. Mathematika 10, 157-164.

[18] VAN DER Hofstad, R. AND KLOK, M. J. (2003). Performance of DS-CDMA systems with optimal hard-decision parallel interference cancellation. IEEE Trans. Inf. Theory 25, 2918-2940.

[19] VAN DER Hofstad, R. AND KLOK, M. J. (2004). Improving the performance of third-generation wireless communication systems. Adv. Appl. Prob. 36, 1046-1084.

[20] Van der Hofstad, R., Hooghiemstra, G. and Klok, M. J. (2002). Analytical methods for CDMA systems with parallel interference cancellation: the large deviation approach. Wireless Personal Commun. 21, $289-307$.

[21] VAn Der Hofstad, R. Hooghiemstra, G. AND KloK, M. J. (2002). Large deviations for code division multiple access systems. SIAM J. Appl. Math. 62, 1044-1065.

[22] Van der Hofstad, R., Löwe, M. and Vermet, F. (2006). The effect of system load on the existence of bit errors in CDMA with and without parallel interference cancelation. IEEE Trans. Inf. Theory 52, 4733-4741.

[23] Yin, Y. Q., Bai, Z. D. And Krishnaiah, P. R. (1988). On the limit of the largest eigenvalue of the largedimensional sample covariance matrix. Prob. Theory Relat. Fields 78, 509-521. 